Review

\title{
Strong Convergence Theorems for Split Common Fixed Point Problem of Bregman Generalized Asymptotically Nonexpansive Mappings in Banach Spaces
}

\author{
Yusuf Ibrahim* \\ Department of Mathematics, Saádatu Rimi College of Education, Kumbotso Kano, Nigeria
}

\begin{abstract}
In this paper, a new iterative scheme is introduced and also strong convergence theorems for solving split common fixed point problem for uniformly continuous Bregman generalized asymptotically nonexpansive mappings in uniformly convex and uniformly smooth Banach spaces are presented. The results are proved without the assumption of semicompactness property and or Opial condition.
\end{abstract}

Keywords: Split common fixed point problem, Bregman distance, Generalized asymptotically nonexpansive mapping, Strong convergence, uniformly convex and uniformly smooth Banach space, Algorithm

Article History :

Received: 04 April 2019

Received in revised form: 30 April 2019

Accepted for publication: 11 May 2019

Published: 16 May 2019

(C)2019 Journal of the Nigerian Society of Physical Sciences. All rights reserved. Communicated by: T. Latunde

\section{Introduction}

Throughout this paper, $E_{1}$ and $E_{2}$ are uniformly convex and uniformly smooth Banach spaces. Let us make some conventions: we always use $p, q \in(1, \infty)$ as conjugate exponents so that $\frac{1}{p}+\frac{1}{q}=1$, where by $q=\frac{p}{p-1}, p q=p+q,(p-1)(q-1)=1$. Furthermore, for real value $a, b$, we write $a \vee b=\max \{a, b\}$ and $a \wedge b=\min \{a, b\}$ which is to be understood componentwise in case of sequences and pointwise in case of functions.

Throughout this paper, let $p, r \geq 1$, given sequences of nonempty closed convex subsets $\left\{C_{i}\right\}_{i=1}^{p}$ and $\left\{Q_{i}\right\}_{i=1}^{r}$ of $E_{1}$ and $E_{2}$, respectively,

Definition 1.1. [1] Multiple set split feasibility problem (MSSFP)

\footnotetext{
${ }^{*}$ Corresponding author Tel. no: +2348062814778

Email address: danustazz@gmail .com (Yusuf Ibrahim)
}

is formulated as finding a point $x^{*} \in E_{1}$ with the property

$$
x^{*} \in \bigcap_{i=1}^{p} C_{i} \text { and } A x^{*} \in \bigcap_{j=1}^{r} Q_{j} .
$$

Definition 1.2. [2] If in a MSSEP (1) $p=r=1$, we get what is called the split feasibility problem (SFP), which is to find a point $x^{*} \in E$, with the property

$$
x^{*} \in C \text { and } A x^{*} \in Q .
$$

Definition 1.3. [3] Split common fixed point problem (SCFPP) is formulated as finding a point $x^{*} \in E_{1}$, with the property

$$
x^{*} \in \bigcap_{i=1}^{p} F i x\left(U_{i}\right) \text { and } A x^{*} \in \bigcap_{j=1}^{r} F i x\left(T_{j}\right) \text {, }
$$

where each $U_{i}: E_{1} \longrightarrow E_{1}(i=1,2, \cdots p)$ and $T_{j}: E_{2} \longrightarrow E_{2}$ 
$(j=1,2, \cdots r)$ are some (nonlinear) mappings.

Definition 1.4. [3] two-set SCFPP is formulated as finding a point $x^{*} \in E_{1}$, with the property

$$
x^{*} \in \operatorname{Fix}(U) \text { and } A x^{*} \in \operatorname{Fix}(T)
$$

where $U: E_{1} \longrightarrow E_{1}$ and $T: E_{2} \longrightarrow E_{2}$ are (nonlinear) mappings.

As stated in [4], let $\mathrm{E}$ be a Banach space with norm $\|\cdot\|$. Let $\mathrm{C}$ be a nonempty closed convex subset of $\mathrm{E}$ and $E^{*}$ denote the dual space of $\mathrm{E}$. Let $B: C \longrightarrow E^{*}$ be a nonlinear mapping and $F: C \times C \longrightarrow \mathfrak{R}$ be a bifunction. The generalized equilibrium problem is to find $x \in C$ such that

$$
F(x, y)+\langle B x, y-x\rangle \geq 0 \forall y \in C .
$$

Now, let

$$
F(x, y)=f(x, y)+g(x, y)
$$

where $f, g: C \times C \longrightarrow \mathfrak{R}$ are two bifunctions satisfying the following special properties $\left(A_{1}\right)-\left(A_{4}\right),\left(B_{1}\right)-\left(B_{3}\right)$ and $(C)$;

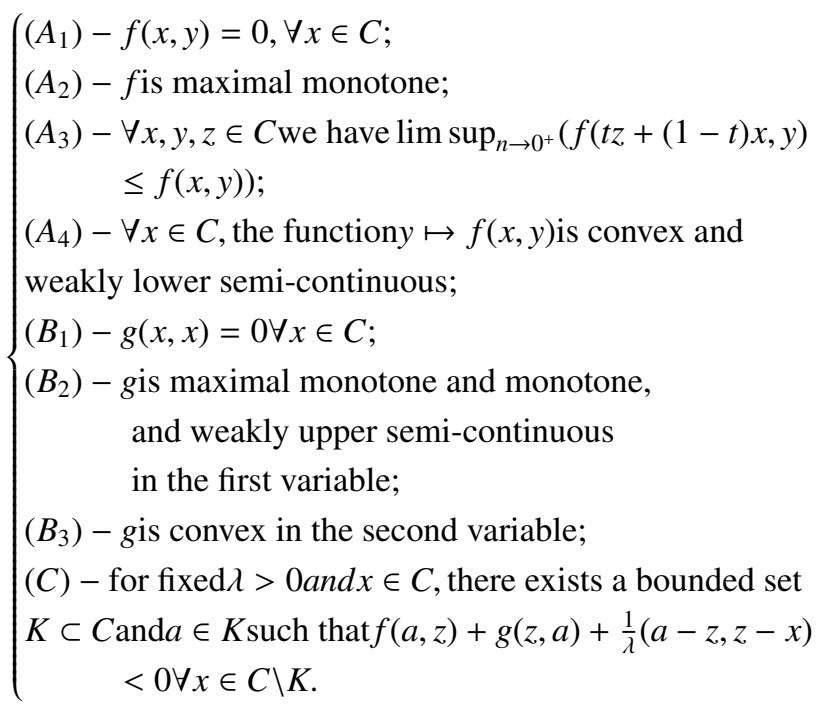

The well known generalized mixed equilibrium problem is to find an $x \in C$ such that

$$
f(x, y)+g(x, y)+\langle B x, y-x\rangle \geq 0 \forall y \in C .
$$

If $B \equiv 0$, the problem (7) reduces into mixed equilibrium problem for $\mathrm{f}$ and $\mathrm{g}$, denoted by $\operatorname{MEP}(f, g)$, which is to find $x \in C$ such that

$$
f(x, y)+g(x, y) \geq 0, \forall y \in C .
$$

If $g \equiv 0$ and $B \equiv 0,(7)$ reduces into equilibrium problem for $\mathrm{f}$, denoted by $\mathrm{EP}(\mathrm{f})$, which is to find $x \in C$ such that

$$
f(x, y) \geq 0 \forall y \in C .
$$

In 1994, the SFP (2) was introduced by Censor and Elfving
[1]. Using the CQ-algorithm for solving the SFP (2), in 2002 Byrne [2] proposed that which generates the new iterate as follows

choosing arbitrarily $x_{1} \in H_{1}$,

$$
x_{n+1}=P_{C}\left[x_{n}-\gamma A^{T}\left(I-P_{Q}\right) A x_{n}\right]
$$

where $\gamma \in\left(0, \frac{2}{L}\right), \mathrm{L}$ denotes the largest eigenvalue of the matrix $A^{T} A$.

In 2004, Yang [5] presented a relaxed CQ-algorithm for solving the SFP, where at n-th iteration, the projections onto $\mathrm{C}$ and $\mathrm{Q}$ were replaced with the halfspaces $C_{n}$ and $Q_{n}$, respectively.

In 2005, Qu and Xiu [6] proposed a modified relaxed CQalgorithm

choosing arbitrarily $x_{1} \in H_{1}$,

$$
x_{n+1}=P_{C_{n}}\left[x_{n}-\alpha_{n} A^{T}\left(I-P_{Q_{n}}\right) A x_{n}\right] .
$$

In 2007, Frank Schopfer[7] developed iterative methods for the solution of the SFP (2) in Banach spaces and also analyse stability and regularizing properties. these iterative methods are as follows.

$$
\begin{array}{r}
\text { choosing arbitrarily } x_{1} \in E_{1}, \\
x_{n+1}=J_{E_{1}}^{*}\left(J_{E_{1}}\left(x_{n}\right)-\mu_{n} A^{*} J_{E_{2}}\left(A x_{n}-P_{Q}\left(A x_{n}\right)\right) .\right.
\end{array}
$$

The concept of SCFPP in finite dimensional Hilbert spaces, say $H_{1}$ and $H_{2}$, was first introduced by Censor and Segal in 2009[1], who invented an algorithm of the two-set SCFPP which generate a sequence $\left\{x_{n}\right\}$ according to the following iterative procedure:

$$
\begin{array}{r}
\text { choosing arbitrarily } x_{1} \in H_{1}, \\
x_{n+1}=U\left(x_{n}+\gamma A^{*}(I-T) A x_{n}\right), n \geq 1
\end{array}
$$

where the initial guess $x_{0} \in H_{1}$ is chosen arbitrarily and $0<$ $\gamma<\frac{1}{\|A\|^{2}}$ and $A: H_{1} \longrightarrow H_{2}$ as a bounded linear operator having $A^{*}$ as the adjoint operator of A. And $U: H_{1} \longrightarrow H_{1}$ and $T: \mathrm{H}_{2} \longrightarrow \mathrm{H}_{2}$ are (nonlinear) mappings.

In 2010, Moudafi [8] proposed the following iteration method to approximate a SCFPP of demicontractive mappings in Hilbert spaces;

$$
\left\{\begin{array}{l}
x_{1} \in H_{1} \text { is arbitrarily chosen, } \\
u_{n}=x_{n}+\gamma A^{*}(I-T) A x_{n}, \\
x_{n+1}=\alpha_{n} u_{n}+\left(1-\alpha_{n}\right) U u_{n},
\end{array}\right.
$$

where he proved that $\left\{x_{n}\right\}$ converges weakly to a split common fixed point $x^{*} \in \Gamma$, where $U: H_{1} \longrightarrow H_{1}$ and $T: H_{2} \longrightarrow H_{2}$ are two demicontractive mappings, $A: H_{1} \longrightarrow H_{2}$ is a bounded linear operator and $H_{1}$ and $H_{2}$ are two Hilbert spaces.

Using the iterative scheme (10), in 2011, Moudafi[8] also obtained a weak convergence theorem for the split common 
fixed poin problem of quasi-nonexpansive mapping in Hilbert spaces.

In 2012, Chang et al. [9] again, using (10) proved the weakly convergence of the sequence $\left\{x_{n}\right\}$ to the split common fixed point $x^{*} \in \Gamma$ of asymptotically quasi-nonexpansive mapping in Hilbert spaces. But these authors could only obtain strong convergence theorem if those mappings and spaces are semi-compacts and Hilbert spaces, respectively.

In 2015, Zhang et al [10] introduced the iterative scheme which guarantees the strong converges for SCFP of the asymptotically nonexpansive mapping in Hilbert spaces, without assumption of semi-compactness. The sequence is defined as follows;

$\left\{\begin{array}{l}\text { choosing arbitrarily } x_{1} \in H_{1}, C_{1}=H_{1}, \\ z_{n}=x_{n}+\lambda A^{*}\left(T_{2}^{n}-I\right) A x_{n}, \\ y_{n}=\alpha_{n} z_{n}+\left(1-\alpha_{n}\right) T_{1}^{n}\left(z_{n}\right), \\ C_{n+1}=\left\{v \in C_{n}:\left\|y_{n}-v\right\| \leq k_{n}\left\|z_{n}-v\right\|,\left\|z_{n}-v\right\| \leq k_{n}\left\|x_{n}-v\right\|\right\}, \\ x_{n+1}=P_{C_{n+1}}\left(x_{1}\right), n \geq 1\end{array}\right.$

where $A^{*}$ denote the adjoint of $\mathrm{A}, \lambda \in\left(0, \frac{1}{\left\|A^{*}\right\|^{2}}\right)$ and $\left\{\alpha_{n}\right\} \subset$ $(0, \tau) \subset(0,1)$ satisfies $\lim _{n \rightarrow \infty} \alpha_{n}\left(1-\alpha_{n}\right)>0, k_{n}=\max \left\{k_{n}^{(1)}, k_{n}^{(2)}\right\}$, $n \geq 1$.

Takahashi [11], in 2015, also obtained a similar result for split common null point problem by using the following hybrid and shrinking projection methods, respectively.

$$
\left\{\begin{array}{l}
\text { choosing arbitrarily } x_{1} \in H_{1}, \\
z_{n}=P_{C}\left(x_{n}-r A^{*} J_{F}\left(A x_{n}-P_{Q} A x_{n}\right)\right), \\
y_{n}=\alpha_{n} x_{n}+\left(1-\alpha_{n}\right) z_{n}, \\
C_{n}=\left\{z \in H_{1}:\left\|y_{n}-z\right\| \leq\left\|x_{n}-z\right\|\right\} \\
Q_{n}=\left\{z \in H_{2}:\left\langle x_{n}-z, x_{1}-x_{n}\right\rangle \geq 0\right\} \\
x_{n+1}=P_{C_{n} \cap Q_{n}} x_{1}, \forall n \in N,
\end{array}\right.
$$

and

$$
\left\{\begin{array}{l}
\text { choosing arbitrarily } x_{1} \in H_{1}, \\
z_{n}=P_{C}\left(x_{n}-r A^{*} J_{F}\left(A x_{n}-P_{Q} A x_{n}\right)\right), \\
C_{n}=\left\{z \in H_{1}:\left\|z_{n}-z\right\| \leq\left\|x_{n}-z\right\|\right\} \cap C_{n}, \\
x_{n+1}=P_{C_{n+1}} u_{n+1}, \forall n \in N,
\end{array}\right.
$$

where $0 \leq \alpha_{n} \leq a<1$ for some $a \in R$ and $0<r\|A\|^{2}<2$.

In 2015, Tang et al. [12] introduced the split common fixed point problem (SCFP) for an asymptotical nonexpansive mapping $S$ and a $\tau$-quasi-pseudocontractive mapping $T$ in the setting of two Banach spaces, $E_{1}$ and $E_{2}$, by using the sequence $\left\{x_{n}\right\}$ defined as follows;

$$
\left\{\begin{array}{l}
\text { choosing arbitrarily } x_{1} \in E_{1}, \\
z_{n}=x_{n}+\gamma J_{1}^{-1} A^{*} J_{2}(T-I) A x_{n}, \\
x_{n+1}=\left(1-\alpha_{n}\right) z_{n}+\alpha_{n} S^{n}\left(z_{n}\right), n \geq 1
\end{array}\right.
$$

where $\left\{\alpha_{n}\right\} \subset(0,1)$ with $\liminf _{n \rightarrow \infty} \alpha_{n}\left(1-\alpha_{n}\right)>0$, $\gamma \subset\left(0, \min \left\{\frac{1-2 k^{2}}{\|A\|^{2}}, \frac{1-\tau}{\|A\|^{2}}\right\}\right), L=\sup _{n \geq 1}\left\{l_{n}\right\}$ and $\sum_{n=1}^{\infty}\left(l_{n}-1\right)<\infty$.

In this paper, the modified algorithm of Zhang et al. for the solution of SCFP in Hilbert spaces is studied. Hence, the Bregman generalized asymptotically nonexpansive mapping is used to obtain the strong convergence for SCFPP (6) in uniformly convex and uniformly smooth Banach spaces, without the assumption of semi-compactness property and or without the assumption of Opial condition.

\section{Preliminaries}

Throughout this paper, the classes of Banach spaces we will deal with are [13];

Definition 2.1. A Banach space $E$ is said to be uniformly convex, if $\delta_{E}(\epsilon)=\inf \left\{1-\left\|\frac{1}{2}(x+y)\right\| ;\|x\|=\|y\|=1,\|x-y\| \geq \epsilon\right.$, where $0 \leq \epsilon \leq 2$ and $\left.0<\delta_{E}(\epsilon) \leq 1\right\}$.

Definition 2.2. A Banach space $E$ is said to be uniformly smooth, if $\lim _{r \rightarrow 0}\left(\frac{\rho_{E}(r)}{r}\right)=0$ where $\rho_{E}(r)=\frac{1}{2} \sup \{\|x+y\|+\|x-y\|-2$ : $\|x\|=1,\|y\| \leq r ; 0 \leq r<\infty$ and $\left.0 \leq \rho_{E}(r)<\infty\right\}$. Moreover,

1. $\rho_{E}$ is continuous, convex and nondecreasing with $\rho_{E}(0)=$ 0 and $\rho_{E}(r) \leq r$

2. The function $r \mapsto \frac{\rho_{E}(r)}{r}$ is nondecreasing and fulfills $\frac{\rho_{E}(r)}{r}>$ 0 for all $r>0$

3. $\lim _{r \rightarrow 0} \frac{\rho_{E}(r)}{r}=0$.

Throughout this paper, some important mappings we will be using are;

Definition 2.3. [14] For each $p>1$, let $g(t)=t^{p-1}$ be a gauge function $g: \mathfrak{R}^{+} \longrightarrow \mathfrak{R}^{+}$such that $g(0)=0$ and $\lim _{n \rightarrow \infty} g(t)=$ $\infty$. we defined the ganeralized daulity map by $J_{p}: E \longrightarrow 2^{E^{*}}$ by

$$
J_{g(t)}=J_{p}(x)=\left\{x^{*} \in E^{*} ;\left\langle x, x^{*}\right\rangle=\|x\|\left\|x^{*}\right\|,\left\|x^{*}\right\|=g(\|x\|)=\|x\|^{p-1}\right\} .
$$

The ganeralized daulity mapping has the following basic properties [7].

Lemma 2.1. For every $x \in E$ the set $J_{E}^{p}(x)$ is not empty and convex.

Lemma 2.2. $J_{E}^{p}(x)$ is homogeneous of degree $p-1$, i.e.

$$
J_{E}^{p}(\lambda x)=|\lambda|^{p-1} \operatorname{sgn}(\lambda) J_{E}^{p}(x) \forall x \in E, \lambda \in \mathfrak{R} .
$$

Lemma 2.3. If $J_{E^{*}}^{p}(x)$ is the duality mapping of $E^{*}$ with gauge function $t \mapsto t^{q-1}$ then $x^{*} \in J_{E}^{p}(x)$ iff $x \in J_{E^{*}}^{q}\left(x^{*}\right)$.

Lemma 2.4. [7] In smooth Banach space, the Bregman distance of $x$ to $y$ with respect to the function $f(x)=\frac{1}{p}\|x\|^{p}$ is defined by

$$
\triangle_{p}(x, y)=\frac{1}{q}\|x\|^{p}-\left\langle J^{p}(x), y\right\rangle+\frac{1}{p}\|y\|^{p} .
$$

Definition 2.4. Let $E$ be a smooth Banach space. Let $\triangle_{p}$ be a Bregman distance. A mapping $T: E \longrightarrow E$ is said to be a Bregman generalized asymptotically nonexpansive with $\left\{k_{n}\right\}$ and $\left\{\mu_{n}\right\}$ if there exists nonnegative real sequences $\left\{k_{n}\right\}$ and $\left\{\mu_{n}\right\}$ with $\lim _{n \rightarrow \infty} k_{n}=0$ and $\lim _{n \rightarrow \infty} \mu_{n}=0$ such that $\triangle_{p}\left(T^{n}(x), T^{n}(y)\right)$ $\leq k_{n} \Delta_{p}(x, y)+\triangle_{p}(x, y)+\mu_{n} \forall(x, y) \in E \times E$. 
Definition 2.5. [15] Let $E$ be a reflexive, strictly convex and smooth Banach space. Then for every closed convex subset $C \subset$ $E$ and $x \in E$ there exists a unique element $P_{C}(x) \in C$ such that $\left\|x-P_{C}(x)\right\|=\min _{y \in C}\|x-y\|$ is called the metric projection of $x$ onto C. Moreover, if $J^{p}$ is a duality mapping of $E$, then $x_{0} \in C$ is the metric projection of $x$ onto $C$ iff

$$
\left\langle J^{p}\left(x_{0}-x\right), y-x_{0}\right\rangle \geq 0 \forall y \in C .
$$

Definition 2.6. [7] Let $E$ be a reflexive, strictly convex and smooth Banach space and $J^{p}$ be a duality mapping of $E$. Then for every closed convex subset $C \subset E$ and $x \in E$ there exists a unique element $\Pi_{C}^{p}(x) \in C$ such that $\Delta_{p}\left(x, \Pi_{C}^{p}(x)\right)=$ $\min _{y \in C} \Delta_{p}(x, y) . \quad \Pi_{C}^{p}(x)$ is called the Bregman projection of $x$ onto $C$, with respect to the function $f(x)=\frac{1}{p}\|x\|^{p}$. Moreover $x_{0} \in C$ is the Bregman projection of $x$ onto $C$ iff

$$
\left\langle J^{p}\left(x_{0}\right)-J^{p}(x), y-x_{0}\right\rangle \geq 0
$$

or equivalently

$$
\Delta_{p}\left(x_{0}, y\right) \leq \Delta_{p}(x, y)-\Delta_{p}\left(x, x_{0}\right) \text { for every } y \in C .
$$

Lemma 2.5. [7] The Bregman projection and the metric projection are related via $P_{C}(x)-x=\Pi_{C-x}^{p}(0), \forall x \in E$. Especially we have $P_{C}(0)=\Pi_{C}^{p}(0)$ and thus $\left\|\Pi_{C}^{p}(0)\right\|=\min _{y \in C}\|y\|$.

The Bregman distance has the following properties [7].

Lemma 2.6. $\forall x, y \in E$ and $\left\{x_{n}\right\} \in E, \Delta_{p}(x, y) \geq 0$ and $\triangle_{p}(x, y)=$ $0 \Leftrightarrow x=y$.

Lemma 2.7. $\forall x, y \in E$ and $\lambda \geq 0 \Delta_{p}(-x,-y)=\Delta_{p}(x, y)$ and $\Delta_{p}$ is positively homogeneous of degree $p$, i.e. $\Delta_{p}(\lambda x, \lambda y)=$ $\lambda^{p} \Delta_{p}(x, y)$.

Lemma 2.8. $\Delta_{p}$ is continuous in both arguments and it is strictly convex, weakly lower semicontinuous and Gateaux differentiable with respect to the second variable with derivative $\frac{\partial}{\partial y} \Delta_{p}(x, y)=$ $J^{p}(y)-J^{p}(x)$

Lemma 2.9. $\Delta_{p}$ satisfies the Three-point property that generalizes the "Law of cosines":

$$
\triangle_{p}(x, y)=\Delta_{p}(x, z)+\Delta_{p}(z, y)-\left\langle x-z, J^{p} y-J^{p} z\right\rangle
$$

Lemma 2.10. Let $X$ be reflexive, smooth and strictly convex. Then for all $x, y \in X$ the following holds:

$$
\triangle_{p}(x, y)+\triangle_{p}(y, x)=\left\langle J^{p}(x)-J^{p}(y), x-y\right\rangle
$$

Lemma 2.11. Consider the following assertions;

$$
\begin{aligned}
& \text { 1. } \lim _{n \rightarrow \infty}\left\|x_{n}-x\right\|=0 \\
& \text { 2. } \lim _{n \rightarrow \infty}\left\|x_{n}\right\|=\|x\| \text { and } \lim _{n \rightarrow \infty}\left\langle J^{p}\left(x_{n}\right), x\right\rangle=\left\langle J^{p}(x), x\right\rangle \\
& \text { 3. } \lim _{n \rightarrow \infty} \triangle_{p}\left(x_{n}, x\right)=0 .
\end{aligned}
$$

The implication (1) $\Longrightarrow(2) \Longrightarrow(3)$ are valid. If $E$ is uniformly convex then the assertions are equivalent.
Lemma 2.12. Let us write $\triangle_{q}^{*}\left(x^{*}, y^{*}\right)=\frac{1}{p}\left\|x^{*}\right\|_{E^{*}}^{q}-\left\langle J_{E^{*}}^{q}, y^{*}\right\rangle+$ $\frac{1}{q}\left\|y^{*}\right\|_{E^{*}}^{q}$ for the Bregman distance on the dual space $E^{*}$ with respect to the function $f^{*}\left(x^{*}\right)=\frac{1}{q}\left\|x^{*}\right\|_{E^{*}}^{q}$. Then we have

$$
\begin{gathered}
\triangle_{p}(x, y)=\triangle_{q}^{*}\left(x^{*}, y^{*}\right) . \text { for } x^{*}=J_{E}^{p}(x) \\
\Leftrightarrow J_{E^{*}}^{q}\left(x^{*}\right)=x \text { and } y^{*}=J_{E}^{p}(y)=y \\
\Leftrightarrow J_{E^{*}}^{q}\left(y^{*}\right)=y .
\end{gathered}
$$

Lemma 2.13. $\prod_{C}^{p}(x)$ maps bounded sets onto bounded sets; more precisely we have

$$
\left\|\Pi_{C}^{p}(x)\right\| \leq\left(2^{q-1}\|x\|\right) \vee\left(3\left\|\Pi_{C}^{p}(0)\right\|\right) \forall x \in E .
$$

Throughout this paper, some important characteristic inequalities we will be using are;

Lemma 2.14. [16] In the case of uniformly convex space E, with the duality map $J^{p}$ of $E, \forall x, y \in E$ we have

$$
\begin{aligned}
\|x-y\|^{p} & =\left\langle J^{p}(x-y), x-y\right\rangle \\
& \geq\|x\|^{p}-p\left\langle J^{p}(x), y\right\rangle+\sigma_{p}(x, y) .
\end{aligned}
$$

with

$$
\sigma_{p}(x, y)=p K_{p} \int_{0}^{1} \frac{(\|x-t y\| \vee\|x\|)^{p}}{t} \lambda_{E}\left(\frac{t\|y\|}{2(\|x-t y\| \vee\|x\|)}\right) d t
$$

where by

$$
\begin{aligned}
K_{p}= & 4(2+\sqrt{4}) \min \left\{\frac{1}{2} p(p-1) \wedge 1,\left(\frac{1}{2} p \wedge 1\right)(p-1),\right. \\
& \left.(p-1)\left(1-(\sqrt{3}-1)^{q}\right), 1-(1+(2-\sqrt{3}) q)^{1-p}\right\} .
\end{aligned}
$$

Lemma 2.15. [16] In the case of uniformly smooth space, E, with the duality map $J^{p}$ of $E, \forall x, y \in E$ we have

$$
\begin{aligned}
\|x-y\|^{p} & =\left\langle J^{p}(x-y), x-y\right\rangle \\
& \leq\|x\|^{p}-p\left\langle J^{p}(x), y\right\rangle+\bar{\sigma}_{p}(x, y) .
\end{aligned}
$$

with

$$
\overline{\sigma_{p}}(x, y)=p G_{p} \int_{0}^{1} \frac{(\|x-t y\| \vee\|x\|)^{p}}{t} \rho_{E}\left(\frac{t\|y\|}{2(\|x-t y\| \vee\|x\|)}\right) d t
$$

where by

$$
G_{p}=8 \vee 64 c K_{p}^{-1}
$$

with

$$
\begin{aligned}
K_{p}= & 4(2+\sqrt{4}) \min \left\{\frac{1}{2} p(p-1) \wedge 1,\left(\frac{1}{2} p \wedge 1\right)(p-1),\right. \\
& \left.(p-1)\left(1-(\sqrt{3}-1)^{q}\right), 1-(1+(2-\sqrt{3}) q)^{1-p}\right\}
\end{aligned}
$$

and

$$
c=4 \frac{\lambda_{0}}{\sqrt{1+\lambda_{0}^{2}}-1} \prod_{j=1}^{\infty}\left(1+\frac{15}{2^{j+2}} \lambda_{0}\right)
$$


with

$$
\lambda_{0}=\frac{\sqrt{339}-18}{30} .
$$

Lemma 2.16. [17] Let $E$ be a real uniformly convex Banach space. For arbitrary $r>1$, let $B_{r}(0)=\{x \in E:\|x\| \leq r\}$. Then, there exists a continuous strictly increasing convex function

$$
g:[0, \infty) \longrightarrow[0, \infty), g(0)=0
$$

such that for every $x, y \in B_{r}(0), f_{x} \in J_{p}(x), f_{y} \in J_{p}(y)$, the following inequality hold:

$\|\lambda x+(1-\lambda) y\|^{p} \leq \lambda\|x\|^{p}+(1-\lambda)\|y\|^{p}-\left(\lambda^{p}(1-\lambda)+(1-\lambda)^{p} \lambda\right) g(\|x-y\|)$

and,

$$
\left\langle x-y, f_{x}-f_{y}\right\rangle \geq g(\|x-y\|) .
$$

Lemma 2.17. [17] Let $E$ be a real uniformly smooth Banach space. For arbitrary $r>1$, let $B_{r}(0)=\{x \in E:\|x\| \leq r\}$. Then, there exists a continuous strictly increasing convex function

$$
g:[0, \infty) \longrightarrow[0, \infty), g(0)=0
$$

such that for every $x, y \in B_{r}(0), f_{x} \in J_{q}(x), f_{y} \in J_{q}(y)$, the following inequality hold:

$\|\lambda x+(1-\lambda) y\|^{q} \geq \lambda\|x\|^{q}+(1-\lambda)\|y\|^{q}-\left(\lambda^{q}(1-\lambda)+(1-\lambda)^{q} \lambda\right) g(\|x-y\|)$

and,

$$
\left\langle x-y, f_{x}-f_{y}\right\rangle \leq g(\|x-y\|) .
$$

\section{Main results}

Theorem 3.1. Let $E_{1}$ and $E_{2}$ be two uniformly convex and uniformly smooth Banach spaces, $A: E_{1} \longrightarrow E_{2}$ is bounded and linear operator such that $A(C)$, for $C \subset E_{1}$, is closed and convex, $U: E_{1} \longrightarrow E_{1}$ be a uniformly continuous Bregman generalized asymptotically nonexpansive operator with the sequences $\left\{k_{n}^{(1)}\right\} \subset[0, \infty)$ and $\left\{\mu_{n}^{(1)}\right\} \subset[0, \infty)$ satisfying $\lim _{n \rightarrow \infty} k_{n}^{(1)}=$ 0 and $\lim _{n \rightarrow \infty} \mu_{n}^{(1)}=0$, respectively, and $T: E_{2} \longrightarrow E_{2}$ be a uniformly continuous Bregman generalized asymptotically nonexpansive operator with the sequences $\left\{k_{n}^{(2)}\right\} \subset[0, \infty)$ and $\left\{\mu_{n}^{(2)}\right\} \subset$ $[0, \infty)$ satisfying $\lim _{n \rightarrow \infty} k_{n}^{(2)}=0$ and $\lim _{n \rightarrow \infty} \mu_{n}^{(2)}=0$, respectively, and, for $p, q \in(1, \infty), \Pi_{A C}^{p}: E_{2} \longrightarrow A C$ be a Bregman projection onto a subset $A C$, and $\operatorname{Fix}(U) \neq \phi$ and $F i x\left(\Pi_{A C}^{p} T\right) \neq \phi$ respectively, and $(I-U)$ and $\left(I-\Pi_{A C}^{p} T\right)$ be demiclosed at zero. Let $x_{1} \in E_{1}$ be chosen arbitrarily and let $C_{1}=E_{1}$ and the sequence $\left\{x_{n}\right\}$ be defined as follows;

$$
\left\{\begin{array}{l}
z_{n}=J_{E_{1}^{*}}^{q}\left(J_{E_{1}}^{p} x_{n}-\lambda_{n} A^{*} J_{E_{2}}^{p}\left(I-\Pi_{A C_{n}}^{p} T^{n}\right) A x_{n}\right), \\
y_{n}=J_{E_{1}^{*}}^{q}\left(\alpha_{n} J_{E_{1}}^{p}\left(z_{n}\right)+\left(1-\alpha_{n}\right) J_{E_{1}}^{p}\left(U^{n}\left(z_{n}\right)\right)\right), \\
C_{n+1}=\left\{v \in C_{n}: \triangle_{p}\left(y_{n}, v\right) \leq\left[k_{n}+1\right] \triangle_{p}\left(z_{n}, v\right)+\mu_{n}\right. \\
\left.\triangle_{p}\left(z_{n}, v\right) \leq \triangle_{p}\left(x_{n}, v\right)\right\}, \\
x_{n+1}=\Pi_{C_{n+1}}^{p}\left(x_{1}\right), n \geq 1
\end{array}\right.
$$

where

$$
\lambda_{n}=\left\{\begin{array}{l}
\frac{1}{\|A\|} \frac{1}{\left\|J_{E_{2}}^{p}\left(I-\Pi_{A C_{n}}^{p} T^{n}\right) A x_{n}\right\|}, x_{n} \neq 0 \\
\frac{1}{\|A\|^{p}} \frac{\left\langle J_{E_{2}}^{p}\left(I-\Pi_{A C_{n}}^{p} T^{n}\right) A x_{n}, A x_{n}-\Pi_{A C_{n}}^{p} T^{n} A x_{n}\right\rangle^{p-1}}{\left\|J_{E_{2}}^{p}\left(I-\Pi_{A C_{n}}^{p} T^{n}\right) A x_{n}\right\|^{p}}, x_{n}=0
\end{array}\right.
$$

and $\gamma \in(0,1)$ and $\tau_{n}=\frac{1}{\left\|x_{n}\right\|^{p-1}}$ are chosen such that

$$
\rho_{E_{1}^{*}}\left(\tau_{n}\right)=\frac{\gamma}{2^{q} G_{q}\|A\|} \times \frac{\left\langle J_{E_{2}}^{p}\left(I-\Pi_{A C_{n}}^{p} T^{n}\right) A x_{n}, A x_{n}-\Pi_{A C_{n}}^{p} T^{n} A x_{n}\right\rangle}{\left\|x_{n}\right\|^{p}\left\|J_{E_{2}}^{p}\left(I-\Pi_{A C_{n}}^{p} T^{n}\right) A x_{n}\right\|},
$$

and $A^{*}$ denote the adjoint of $A$, and $\left\{\alpha_{n}\right\} \subset(0,1)$ satisfies $\liminf _{n \rightarrow \infty} \alpha_{n}\left(1-\alpha_{n}\right)>0, k_{n}=\left(k_{n}^{(1)} \vee k_{n}^{(2)}\right), n \geq 1$. If $\Gamma=\{v \in$ $\left.\operatorname{Fix}(U): A v \in F i x\left(\Pi_{A C}^{p} T\right)\right\} \neq \phi$, then $\left\{x_{n}\right\}$ converges strongly to $x^{*} \in \Gamma$.

\section{Proof:}

Assume that $\left\|\lambda_{n} A^{*}\left(J_{E_{2}}^{p}\left(I-\Pi_{A C_{n}}^{p} T^{n}\right) A x_{n}\right)\right\|=0 \Leftrightarrow x_{n} \in \Gamma=$ $\left\{x_{n} \in \operatorname{Fix}(U): A x_{n} \in \operatorname{Fix}\left(\Pi_{A C}^{p} T\right)\right\} \neq \phi$ then from 11 we have that $z_{n}=x_{n}$ and $\Delta_{p}\left(x_{n}, z_{n}\right)=0$ and the conclusion follows immediately.

Now assume that $\left\|\lambda_{n} A^{*}\left(J_{E_{2}}^{p}\left(I-\Pi_{A C_{n}}^{p} T^{n}\right) A x_{n}\right)\right\| \neq 0$ then we will divide the proof into five steps.

Step one: We first show that $C_{n}$ is closed and convex, for any $n \geq 1$.

By induction hypothesis; since $C_{1}=E_{1}$, so $C_{1}$ is closed and convex.

Assuming that $C_{k}$ is closed and convex.

We show that $C_{k+1}$ is closed and convex, for some $k \geq 0$.

We first show that $C_{k+1}$ is closed.

Let $\left\{x_{m}\right\}$ be a sequence in $C_{k+1}$ such that $x_{m} \rightarrow v$ as $n \rightarrow \infty$. We need to show that $v \in C_{k+1}$. From 11 and continuity of Bregman distance and the boundedness of $\left\{x_{m}\right\}$, we have that

$$
\begin{aligned}
\triangle_{p}\left(z_{n}, v\right) & =\lim _{m \rightarrow \infty} \triangle_{p}\left(z_{n}, x_{m}\right) \\
& \leq \lim _{m \rightarrow \infty} \triangle_{p}\left(x_{n}, x_{m}\right)=\triangle_{p}\left(x_{n}, v\right) .
\end{aligned}
$$

Similarly, we have that

$$
\begin{aligned}
& \Delta_{p}\left(y_{n}, v\right)=\lim _{m \rightarrow \infty} \Delta_{p}\left(y_{n}, x_{m}\right) \\
& \quad \leq \lim _{m \rightarrow \infty}\left(\left[k_{n}+1\right] \triangle_{p}\left(z_{n}, x_{m}\right)+\mu_{n}\right)=\left[k_{n}+1\right]\left(\triangle_{p}\left(x_{n}, v\right)+\mu_{n} .\right.
\end{aligned}
$$

From (14) and (15), we have that $v \in C_{k+1}$.

Next we show that $C_{k+1}$ is convex.

Let $v_{1}, v_{2} \in C_{k+1}$ and $t \in(0,1)$; putting $v=t v_{1}+(1-t) v_{2}$, it suffices to show that $v \in C_{k+1}$. Let $x_{k} \neq 0$, then

$$
\begin{aligned}
& \triangle_{p}\left(z_{k}, v\right)=\triangle_{p}\left(z_{k}, t v_{1}+(1-t) v_{2}\right) \\
& \quad=\frac{1}{q}\left\|J_{E_{1}}^{p} x_{k}-\lambda_{k} A^{*}\left(J_{E_{2}}^{p}\left(I-\Pi_{A C_{k}}^{p} T^{k}\right) A x_{k}\right)\right\|^{q}+\frac{1}{p}\left\|t v_{1}+(1-t) v_{2}\right\|^{p} \\
& \quad-\left\langle J_{E_{1}}^{p} x_{k}-\lambda_{k} A^{*}\left(J_{E_{2}}^{p}\left(I-\Pi_{A C_{k}}^{p} T^{k}\right) A x_{k}\right), t v_{1}+(1-t) v_{2}\right\rangle
\end{aligned}
$$




$$
\begin{aligned}
& =\frac{1}{q} \| J_{E_{1}}^{p} x_{k}-\lambda_{k} A^{*}\left(J_{E_{2}}^{p}\left(I-\Pi_{A C_{k}}^{p} T^{k}\right) A x_{k}\left\|^{q}+\frac{1}{p}\right\| t v_{1}+(1-t) v_{2} \|^{p}\right. \\
& -\left\langle J_{E_{1}}^{p} x_{k}, t v_{1}+(1-t) v_{2}\right\rangle+\left\langle\lambda _ { k } \left( J_{E_{2}}^{p}\left(I-\Pi_{A C_{k}}^{p} T^{k}\right) A x_{k}, t v_{1}\right.\right. \\
& \left.+(1-t) v_{2}\right\rangle .
\end{aligned}
$$$$
=\frac{1}{q}\left\|x_{k}\right\|^{p}-\lambda_{k}\left\langle A x_{k}, J_{E_{2}}^{p}\left(I-\Pi_{A C_{k}}^{p} T^{k}\right) A x_{k}\right\rangle
$$$$
+\frac{1}{q} \bar{\sigma}_{q}\left(J_{E_{1}}^{p} x_{k}, \lambda_{k} A^{*} J_{E_{2}}^{p}\left(I-\Pi_{A C_{k}}^{p} T^{k}\right) A x_{k}\right)
$$

Where

$$
\begin{aligned}
& \lambda_{k}\left\langle A^{*}\left(J_{E_{2}}^{p}\left(I-\Pi_{A C_{k}}^{p} T^{k}\right) A x_{k}, t v_{1}+(1-t) v_{2}\right\rangle\right. \\
& =\lambda_{k}\left\langle\left(J_{E_{2}}^{p}\left(I-\Pi_{A C_{k}}^{p} T^{k}\right) A x_{k}, t A v_{1}+(1-t) A v_{2}+\Pi_{A C_{k}}^{p} T^{k} A x_{k}-\Pi_{A C_{k}}^{p} T^{k} A x_{k}\right\rangle\right. \\
& =\lambda_{k}\left\langle\left(J_{E_{2}}^{p}\left(I-\Pi_{A C_{k}}^{p} T^{k}\right) A x_{k}, t A v_{1}+(1-t) A v_{2}-\Pi_{A C_{k}}^{p} T^{k} A x_{k}\right\rangle\right. \\
& +\lambda_{k}\left\langle\left(J_{E_{2}}^{p}\left(I-\Pi_{A C_{k}}^{p} T^{k}\right) A x_{k}, \Pi_{A C_{k}}^{p} T^{k} A x_{k}\right\rangle\right. \\
& =\lambda_{k}\left\langle\left(J_{E_{2}}^{p}\left(I-\Pi_{A C_{k}}^{p} T^{k}\right) A x_{k}, t A v_{1}+(1-t) A v_{2}-\Pi_{A C_{k}}^{p} T^{k} A x_{k}\right\rangle\right. \\
& +\lambda_{k}\left\langle\left(J_{E_{2}}^{p}\left(I-\Pi_{A C_{k}}^{p} T^{k}\right) A x_{k}, \Pi_{A C_{k}}^{p} T^{k} A x_{k}-A x_{k}+A x_{k}\right\rangle\right. \\
& =\lambda_{k}\left\langle\left(J_{E_{2}}^{p}\left(I-\Pi_{A C_{k}}^{p} T^{k}\right) A x_{k}, t A v_{1}+(1-t) A v_{2}-\Pi_{A C_{k}}^{p} T^{k} A x_{k}\right\rangle\right. \\
& -\lambda_{k}\left\langle\left(J_{E_{2}}^{p}\left(I-\Pi_{A C_{k}}^{p} T^{k}\right) A x_{k}, \Pi_{A C_{k}}^{p} T^{k} A x_{k}-A x_{k}\right\rangle\right. \\
& +\lambda_{k}\left\langle\left(J_{E_{2}}^{p}\left(I-\Pi_{A C_{k}}^{p} T^{k}\right) A x_{k}, A x_{k}\right\rangle\right. \\
& =\lambda_{k}\left\langle\left(J_{E_{2}}^{p}\left(I-\Pi_{A C_{k}}^{p} T^{k}\right) A x_{k}, t A v_{1}+(1-t) A v_{2}-A x_{k}+A x_{k}-\Pi_{A C_{k}}^{p} T^{k} A x_{k}\right\rangle\right. \\
& -\lambda_{k}\left\langle\left(J_{E_{2}}^{p}\left(I-\Pi_{A C_{k}}^{p} T^{k}\right) A x_{k}, A x_{k}-\Pi_{A C_{k}}^{p} T^{k} A x_{k}\right\rangle\right. \\
& +\lambda_{k}\left\langle\left(J_{E_{2}}^{p}\left(I-\Pi_{A C_{k}}^{p} T^{k}\right) A x_{k}, A x_{k}\right\rangle\right. \\
& =\lambda_{k}\left\langle\left( J_{E_{2}}^{p}\left(I-\Pi_{A C_{k}}^{p} T^{k}\right) A x_{k},\left(t A v_{1}+(1-t) A v_{2}-A x_{k}\right)\right.\right. \\
& \left.-\left(\Pi_{A C_{k}}^{p} T^{k} A x_{k}-A x_{k}\right)\right\rangle-\lambda_{k}\left\langle\left(J_{E_{2}}^{p}\left(I-\Pi_{A C_{k}}^{p} T^{k}\right) A x_{k}, A x_{k}-\Pi_{A C_{k}}^{p} T^{k} A x_{k}\right\rangle\right. \\
& +\lambda_{k}\left\langle\left(J_{E_{2}}^{p}\left(I-\Pi_{A C_{k}}^{p} T^{k}\right) A x_{k}, A x_{k}\right\rangle\right. \\
& =-\lambda_{k}\left\langle\left( J_{E_{2}}^{p}\left(\Pi_{A C_{k}}^{p} T^{k}-I\right) A x_{k},\left(t A v_{1}+(1-t) A v_{2}-A x_{k}\right)\right.\right. \\
& \left.-\left(\Pi_{A C_{k}}^{p} T^{k} A x_{k}-A x_{k}\right)\right\rangle-\lambda_{k}\left\langle\left(J_{E_{2}}^{p}\left(I-\Pi_{A C_{k}}^{p} T^{k}\right) A x_{k}, A x_{k}-\Pi_{A C_{k}}^{p} T^{k} A x_{k}\right\rangle\right. \\
& +\lambda_{k}\left\langle\left(J_{E_{2}}^{p}\left(I-\Pi_{A C_{k}}^{p} T^{k}\right) A x_{k}, A x_{k}\right\rangle .\right. \\
&
\end{aligned}
$$

By the assumption that $A\left(C_{k}\right)$ is closed and convex and Lemma 2.5 and by the variational inequality for the Bregman projection of zero onto $A\left(C_{k}\right)-A x_{k}$, as in Definition 2.6, we arrive at

$$
\begin{aligned}
& \left\langle\left( J_{E_{2}}^{p}\left(\Pi_{A C_{k}}^{p} T^{k}-I\right) A x_{k},\left(t A v_{1}+(1-t) A v_{2}-A x_{k}\right)\right.\right. \\
& \left.-\left(\Pi_{A C_{k}}^{p} T^{k} A x_{k}-A x_{k}\right)\right\rangle \geq 0
\end{aligned}
$$

and therefore,

$$
\begin{aligned}
\lambda_{k} & \left\langle J_{E_{2}}^{p}\left(I-\Pi_{A C_{k}}^{p} T^{k}\right) A x_{k}, t A v_{1}+(1-t) A v_{2}\right\rangle \\
& \leq \lambda_{k}\left\langle J_{E_{2}}^{p}\left(I-\Pi_{A C_{k}}^{p} T^{k}\right) A x_{k}, A x_{k}\right\rangle \\
& -\lambda_{k}\left\langle J_{E_{2}}^{p}\left(I-\Pi_{A C_{k}}^{p} T^{k}\right) A x_{k}, A x_{k}-\Pi_{A C_{k}}^{p} T^{k} A x_{k}\right\rangle .
\end{aligned}
$$

In addition, from Lemma 2.15, we have that

$$
\begin{aligned}
\frac{1}{q} & \| J_{E_{1}}^{p} x_{k}-\lambda_{k} A^{*}\left(J_{E_{2}}^{p}\left(I-\Pi_{A C_{k}}^{p} T^{k}\right) A x_{k} \|^{q}\right. \\
& \leq \frac{1}{q}\left\|J_{E_{1}}^{p} x_{k}\right\|^{q}-\lambda_{k}\left\langle x_{k}, A^{*} J_{E_{2}}^{p}\left(I-\Pi_{A C_{k}}^{p} T^{k}\right) A x_{k}\right\rangle \\
& +\frac{1}{q} \overline{\sigma_{q}}\left(J_{E_{1}}^{p} x_{k}, \lambda_{k} A^{*} J_{E_{2}}^{p}\left(I-\Pi_{A C_{k}}^{p} T^{k}\right) A x_{k}\right) \\
& =\frac{1}{q}\left\|J_{E_{1}}^{p} x_{k}\right\|^{q}-\lambda_{k}\left\langle A x_{k}, J_{E_{2}}^{p}\left(I-\Pi_{A C_{k}}^{p} T^{k}\right) A x_{k}\right\rangle \\
& +\frac{1}{q} \bar{\sigma}_{q}\left(J_{E_{1}}^{p} x_{k}, \lambda_{k} A^{*} J_{E_{2}}^{p}\left(I-\Pi_{A C_{k}}^{p} T^{k}\right) A x_{k}\right)
\end{aligned}
$$

and

$$
\begin{aligned}
& \frac{1}{q} \bar{\sigma}_{q}\left(J_{E_{1}}^{p} x_{k}, \lambda_{k} A^{*} J_{E_{2}}^{p}\left(I-\Pi_{A C_{k}}^{p} T^{k}\right) A x_{k}\right) \\
& \quad=G_{q} \int_{0}^{1} \frac{\left(\left\|J_{E_{1}}^{p} x_{k}-t \lambda_{k} A^{*} J_{E_{2}}^{p}\left(I-\Pi_{A C_{k}}^{p} T^{k}\right) A x_{k}\right\| \vee\left\|J_{E_{1}}^{p} x_{k}\right\|\right)^{q}}{t} \times \\
& \rho_{E^{*}}\left(\frac{t\left\|\lambda_{k} A^{*} J_{E_{2}}^{p}\left(I-\Pi_{A C_{k}}^{p} T^{k}\right) A x_{k}\right\|}{\left(\left\|J_{E_{1}}^{p} x_{k}-t \lambda_{k} A^{*} J_{E_{2}}^{p}\left(I-\Pi_{A C_{k}}^{p} T^{k}\right) A x_{k}\right\| \vee\left\|J_{E_{1}}^{p} x_{k}\right\|\right)}\right) d t,
\end{aligned}
$$

for every $t \in[0,1]$.

But

$$
\begin{aligned}
& \left\|J_{E_{1}}^{p} x_{k}-t \lambda_{k} A^{*} J_{E_{2}}^{p}\left(I-\Pi_{A C_{k}}^{p} T^{k}\right) A x_{k}\right\| \\
& \leq\left\|x_{k}\right\|^{p-1}+\left\|\lambda_{k} A^{*} J_{E_{2}}^{p}\left(I-\Pi_{A C_{k}}^{p} T^{k}\right) A x_{k}\right\| .
\end{aligned}
$$

From (12), suppose that

$$
\lambda_{k}=\frac{\tau_{k}}{\|A\|} \frac{\left\|x_{k}\right\|^{p-1}}{\left\|J_{E_{2}}^{p}\left(I-\Pi_{A C_{n}}^{p} T^{n}\right) A x_{n}\right\|}
$$

then we have that

$$
\left\|J_{E_{1}}^{p} x_{k}-t \lambda_{k} A^{*} J_{E_{2}}^{p}\left(I-\Pi_{A C_{k}}^{p} T^{k}\right) A x_{k}\right\| \leq 2\left\|x_{k}\right\|^{p-1}
$$

and that

$\left\|x_{k}\right\|^{p-1} \leq\left\|J_{E_{1}}^{p} x_{k}-t \lambda_{k} A^{*} J_{E_{2}}^{p}\left(I-\Pi_{A C_{k}}^{p} T^{k}\right) A x_{k}\right\| \vee\left\|J_{E_{1}}^{p} x_{k}\right\| \leq 2\left\|x_{k}\right\|^{p-1}$.

From Definition 2.2 (2), (12) and (20), we have that

$$
\begin{aligned}
& \rho_{E_{1}^{*}}\left(\frac{t\left\|\lambda_{k} A^{*} J_{E_{2}}^{p}\left(I-\Pi_{A C_{k}}^{p} T^{k}\right) A x_{k}\right\|}{\left(\left\|J_{E_{1}}^{p} x_{k}-t \lambda_{k} A^{*} J_{E_{2}}^{p}\left(I-\Pi_{A C_{k}}^{p} T^{k}\right) A x_{k}\right\| \vee\left\|J_{E_{1}}^{p} x_{k}\right\|\right)}\right) \\
& \quad \leq \rho_{E_{1}^{*}}\left(\frac{t\left\|\lambda_{k} A^{*} J_{E_{2}}^{p}\left(I-\Pi_{A C_{k}}^{p} T^{k}\right) A x_{k}\right\|}{\left\|x_{k}\right\|^{p-1}}\right) \\
& \quad=\rho_{E_{1}^{*}}\left(t \tau_{k}\right) .
\end{aligned}
$$

Substituting Eqs. (20) and (21) into (19), and since $t \leq \tau_{k}$ and $\rho_{E_{1}^{*}}$ is nondecreasing function then, we have that

$$
\begin{aligned}
\frac{1}{q} \bar{\sigma}_{q} & \left(J_{E_{2}}^{p} x_{k}, \lambda_{k} A^{*} J_{E_{2}}^{p}\left(I-\Pi_{A C_{k}}^{p} T^{k}\right) A x_{k}\right) \\
& \leq 2^{q} G_{q}\left\|x_{k}\right\|^{(p-1) q} \int_{0}^{\tau_{k}} \frac{\rho_{E_{1}^{*}}(t)}{t} d t \\
& \leq 2^{q} G_{q}\left\|x_{k}\right\|^{p} \int_{0}^{\tau_{k}} \frac{\rho_{E_{1}^{*}}\left(\tau_{k}\right)}{\tau_{k}} d t \\
& =2^{q} G_{q}\left\|x_{k}\right\|^{p} \rho_{E_{1}^{*}}\left(\tau_{k}\right) .
\end{aligned}
$$


Substituting Eqs. (22) into (18), we have that

$$
\begin{aligned}
& \frac{1}{q}\left\|J_{E_{1}}^{p} x_{k}-\lambda_{k} A^{*} J_{E_{2}}^{p}\left(I-\Pi_{A C_{k}}^{p} T^{k}\right) A x_{k}\right\|^{q} \\
& \quad \leq \frac{1}{q}\left\|x_{k}\right\|^{p}-\lambda_{k}\left\langle A x_{k}, J_{E_{2}}^{p}\left(I-\Pi_{A C_{k}}^{p} T^{k}\right) A x_{k}\right\rangle+2^{q} G_{q}\left\|x_{k}\right\|^{p} \rho_{E_{1}^{*}}\left(\tau_{k}\right) \\
& \quad=\frac{1}{q}\left\|J_{E_{1}}^{p} x_{k}\right\|^{q}-\lambda_{k}\left\langle\left(J_{E_{2}}^{p}\left(I-\Pi_{A C_{k}}^{p} T^{k}\right) A x_{k}, A x_{k}\right\rangle\right. \\
& \quad+2^{q} G_{q}\left\|x_{k}\right\|^{p} \rho_{E_{1}^{*}}\left(\tau_{k}\right) .
\end{aligned}
$$

Substituting (23) and (17) into (16), we have that

$$
\begin{aligned}
\triangle_{p} & \left(z_{k}, t v_{1}+(1-t) v_{2}\right) \leq \frac{1}{q}\left\|x_{k}\right\|^{p}+\frac{1}{p}\left\|t v_{1}+(1-t) v_{2}\right\|^{p} \\
& -\left\langle J_{E_{1}}^{p} x_{k}, t v_{1}+(1-t) v_{2}\right\rangle+2^{q} G_{q}\left\|x_{k}\right\|^{p} \rho_{E_{1}^{*}}\left(\tau_{k}\right) \\
& -\lambda_{n}\left\langle\left(J_{E_{2}}^{p}\left(I-\Pi_{A C_{k}}^{p} T^{k}\right) A x_{k}\right), A x_{k}-\Pi_{A C_{k}}^{p} T^{k} A x_{k}\right\rangle \\
& =\triangle_{p}\left(x_{k}, t v_{1}+(1-t) v_{2}\right)+2^{q} G_{q}\left\|x_{k}\right\|^{p} \rho_{E_{1}^{*}}\left(\tau_{k}\right) \\
& \left.-\lambda_{n}\left\langle\left(J_{E_{2}}^{p}\left(I-\Pi_{A C_{k}}^{p} T^{k}\right) A x_{k}\right), A x_{k}-\Pi_{A C_{k}}^{p} T^{k}\right) A x_{k}\right\rangle
\end{aligned}
$$

Substituting (13) and (12) into (24), we have that

$$
\begin{array}{r}
\triangle_{p}\left(z_{k}, t v_{1}+(1-t) v_{2}\right) \leq \Delta_{p}\left(x_{k}, t v_{1}+(1-t) v_{2}\right) \\
+\frac{\gamma\left\langle J_{E_{2}}^{p}\left(I-\Pi_{A C_{k}}^{p} T^{k}\right) A x_{k}, A x_{k}-\Pi_{A C_{k}}^{p} T^{k} A x_{k}\right\rangle}{\left.\|A\| \| J_{E_{2}}^{p}\left(I-\Pi_{A C_{k}}^{p} T^{k}\right) A x_{k}\right) \|} \\
-\frac{\left.\left\langle J_{E_{2}}^{p}\left(I-\Pi_{A C_{k}}^{p} T^{k}\right) A x_{k}, A x_{k}-\Pi_{A C_{k}}^{p} T^{k}\right) A x_{k}\right\rangle}{\left\|A^{*}\right\|\left\|J_{E_{2}}^{p}\left(I-\Pi_{A C_{k}}^{p} T^{k}\right) A x_{k}\right\|} \\
\quad=\triangle_{p}\left(x_{k}, t v_{1}+(1-t) v_{2}\right)-[1-\gamma] \\
\quad \times \frac{\left.\left\langle J_{E_{2}}^{p}\left(I-\Pi_{A C_{k}}^{p} T^{k}\right) A x_{k}, A x_{k}-\Pi_{A C_{k}}^{p} T^{k}\right) A x_{k}\right\rangle}{\left.\|A\| \| J_{E_{2}}^{p}\left(I-\Pi_{A C_{k}}^{p} T^{k}\right) A x_{k}\right) \|} .
\end{array}
$$

Therefore,

$$
\triangle_{p}\left(z_{k}, t v_{1}+(1-t) v_{2}\right) \leq \triangle_{p}\left(x_{k}, t v_{1}+(1-t) v_{2}\right)
$$

Let $x_{k}=0$, we have

$$
\triangle_{p}\left(x_{k}, t v_{1}+(1-t) v_{2}\right)=\frac{1}{p}\left\|t v_{1}+(1-t) v_{2}\right\|^{p}
$$

and from (28), we have

$$
\begin{aligned}
& \triangle_{p}\left(z_{k}, t v_{1}+(1-t) v_{2}\right)=\frac{1}{q}\left\|\lambda_{k} A^{*} J_{E_{2}}^{p}\left(I-\Pi_{A C_{k}}^{p} T^{k}\right) A x_{k}\right\|^{q} \\
& \quad+\triangle_{p}\left(x_{k}, t v_{1}+(1-t) v_{2}\right) \\
& \left.\quad+\lambda_{k}\left\langle J_{E_{2}}^{p}\left(I-\Pi_{A C_{k}}^{p} T^{k}\right) A x_{k}\right), t A v_{1}+(1-t) A v_{2}\right\rangle .
\end{aligned}
$$

Substituting (17) into (29), we have that

$$
\begin{aligned}
& \triangle_{p}\left(z_{k}, t v_{1}+(1-t) v_{2}\right) \leq \frac{1}{q}\left\|\lambda_{k} A^{*} J_{E_{2}}^{p}\left(I-\Pi_{A C_{k}}^{p} T^{k}\right) A x_{k}\right\|^{q} \\
& \quad+\triangle_{p}\left(x_{k}, t v_{1}+(1-t) v_{2}\right)+\lambda_{n}\left\langle J_{E_{2}}^{p}\left(I-\Pi_{A C_{k}}^{p} T^{k}\right) A x_{k}, A x_{k}\right\rangle
\end{aligned}
$$

$$
\left.-\lambda_{n}\left\langle J_{E_{2}}^{p}\left(I-\Pi_{A C_{k}}^{p} T^{k}\right) A x_{k}, A x_{k}-\Pi_{A C_{k}}^{p} T^{k}\right) A x_{k}\right\rangle .
$$

But, from (12), we have that

$$
\begin{aligned}
& \frac{1}{q}\left\|\lambda_{k} A^{*} J_{E_{2}}^{p}\left(I-\Pi_{A C_{k}}^{p} T^{k}\right) A x_{k}\right\|^{q} \\
& \quad=\frac{1}{q} \frac{1}{\|A\|^{p}} \frac{\left.\left\langle J_{E_{2}}^{p}\left(I-\Pi_{A C_{k}}^{p} T^{k}\right) A x_{k}, A x_{k}-\Pi_{A C_{k}}^{p} T^{k}\right) A x_{k}\right\rangle^{p}}{\left\|J_{E_{2}}^{p}\left(I-\Pi_{A C_{k}}^{p} T^{k}\right) A x_{k}\right\|^{p}} .
\end{aligned}
$$

Substituting (31) into (30), we have that

$$
\begin{aligned}
& \triangle_{p}\left(z_{k}, t v_{1}+(1-t) v_{2}\right) \\
& \leq \frac{1}{q} \frac{1}{\|A\|^{p}} \frac{\left.\left\langle J_{E_{2}}^{p}\left(I-\Pi_{A C_{k}}^{p} T^{k}\right) A x_{k}, A x_{k}-\Pi_{A C_{k}}^{p} T^{k}\right) A x_{k}\right\rangle^{p}}{\left\|J_{E_{2}}^{p}\left(I-\Pi_{A C_{k}}^{p} T^{k}\right) A x_{k}\right\|^{p}} \\
& +\triangle_{p}\left(x_{k}, t v_{1}+(1-t) v_{2}\right)+\lambda_{k}\left\langle J_{E_{2}}^{p}\left(I-\Pi_{A C_{k}}^{p} T^{k}\right) A x_{k}, A x_{k}\right\rangle \\
& \left.-\lambda_{n}\left\langle J_{E_{2}}^{p}\left(I-\Pi_{A C_{k}}^{p} T^{k}\right) A x_{k}, A x_{k}-\Pi_{A C_{k}}^{p} T^{k}\right) A x_{k}\right\rangle \\
& \leq\left(1-\frac{1}{p}\right) \frac{1}{\|A\|^{p}} \frac{\left.\left\langle J_{E_{2}}^{p}\left(I-\Pi_{A C_{k}}^{p} T^{k}\right) A x_{k}, A x_{k}-\Pi_{A C_{k}}^{p} T^{k}\right) A x_{k}\right\rangle^{p}}{\left\|J_{E_{2}}^{p}\left(I-\Pi_{A C_{k}}^{p} T^{k}\right) A x_{k}\right\|^{p}} \\
& +\triangle_{p}\left(x_{k}, t v_{1}+(1-t) v_{2}\right)+\lambda_{k}\left\langle A^{*} J_{E_{2}}^{p}\left(I-\Pi_{A C_{k}}^{p} T^{k}\right) A x_{k}, x_{k}\right\rangle \\
& -\frac{1}{\|A\|^{p}} \frac{\left.\left\langle J_{E_{2}}^{p}\left(I-\Pi_{A C_{k}}^{p} T^{k}\right) A x_{k}, A x_{k}-\Pi_{A C_{k}}^{p} T^{k}\right) A x_{k}\right\rangle^{p}}{\left\|J_{E_{2}}^{p}\left(I-\Pi_{A C_{k}}^{p} T^{k}\right) A x_{k}\right\|^{p}} \\
& \left.\times\left\langle J_{E_{2}}^{p}\left(I-\Pi_{A C_{k}}^{p} T^{k}\right) A x_{k}, A x_{k}-\Pi_{A C_{k}}^{p} T^{k}\right) A x_{k}\right\rangle \\
& \leq\left(1-\frac{1}{p}\right) \frac{1}{\|A\|^{p}} \frac{\left.\left\langle J_{E_{2}}^{p}\left(I-\Pi_{A C_{k}}^{p} T^{k}\right) A x_{k}, A x_{k}-\Pi_{A C_{k}}^{p} T^{k}\right) A x_{k}\right\rangle^{p}}{\left\|J_{E_{2}}^{p}\left(I-\Pi_{A C_{k}}^{p} T^{k}\right) A x_{k}\right\|^{p}} \\
& +\triangle_{p}\left(x_{k}, t v_{1}+(1-t) v_{2}\right)+\lambda_{k}\left\|J_{E_{2}}^{p}\left(I-\Pi_{A C_{k}}^{p} T^{k}\right) A x_{k}\right\|\left\|A x_{k}\right\| \\
& -\frac{1}{\|A\|^{p}} \frac{\left.\left\langle J_{E_{2}}^{p}\left(I-\Pi_{A C_{k}}^{p} T^{k}\right) A x_{k}, A x_{k}-\Pi_{A C_{k}}^{p} T^{k}\right) A x_{k}\right\rangle^{p}}{\left\|J_{E_{2}}^{p}\left(I-\Pi_{A C_{k}}^{p} T^{k}\right) A x_{k}\right\|^{p}} \\
& \left.\times\left\langle J_{E_{2}}^{p}\left(I-\Pi_{A C_{k}}^{p} T^{k}\right) A x_{k}, A x_{k}-\Pi_{A C_{k}}^{p} T^{k}\right) A x_{k}\right\rangle \\
& =\triangle_{p}\left(x_{k}, t v_{1}+(1-t) v_{2}\right) \\
& -\frac{1}{p} \frac{1}{\|A\|^{p}} \frac{\left.\left\langle J_{E_{2}}^{p}\left(I-\Pi_{A C_{k}}^{p} T^{k}\right) A x_{k}, A x_{k}-\Pi_{A C_{k}}^{p} T^{k}\right) A x_{k}\right\rangle^{p}}{\left\|J_{E_{2}}^{p}\left(I-\Pi_{A C_{k}}^{p} T^{k}\right) A x_{k}\right\|^{p}} \text {. }
\end{aligned}
$$

This implies that

$$
\triangle_{p}\left(z_{k}, t v_{1}+(1-t) v_{2}\right) \leq \triangle_{p}\left(x_{k}, t v_{1}+(1-t) v_{2}\right)
$$

In addition, it follows from Eq. (11), Definition 2.4 and Lemma 2.16 that

$$
\begin{aligned}
\Delta_{p} & \left(y_{k}, t v_{1}+(1-t) v_{2}\right) \\
& =\Delta_{p}\left(J_{E_{1}^{*}}^{q}\left(\alpha_{k} J_{E_{1}}^{p}\left(z_{k}\right)+\left(1-\alpha_{k}\right) J_{E_{1}}^{p}\left(U^{k}\left(z_{k}\right)\right), t v_{1}+(1-t) v_{2}\right)\right. \\
& =\frac{1}{q} \| \alpha_{k} J_{E_{1}}^{p}\left(z_{k}\right)+\left(1-\alpha_{k}\right) J_{E_{1}}^{p}\left(U^{k}\left(z_{k}\right) \|^{q}\right. \\
& -\left\langle\alpha_{k} J_{E_{1}}^{p}\left(z_{k}\right)+\left(1-\alpha_{k}\right) J_{E_{1}}^{p}\left(U^{k}\left(z_{k}\right), t v_{1}+(1-t) v_{2}\right\rangle\right. \\
& +\frac{1}{p}\left\|t v_{1}+(1-t) v_{2}\right\|^{p} \\
& =\frac{1}{q} \| \alpha_{k} J_{E_{1}}^{p}\left(z_{k}\right)+\left(1-\alpha_{k}\right) J_{E_{1}}^{p}\left(U^{k}\left(z_{k}\right) \|^{p}-\alpha_{k}\left\langle J_{E_{1}}^{p} z_{k}, t v_{1}+(1-t) v_{2}\right\rangle\right.
\end{aligned}
$$




$$
\begin{aligned}
& -\left(1-\alpha_{k}\right)\left\langle J_{E_{1}}^{p}\left(U^{k}\left(z_{k}\right)\right), t v_{1}+(1-t) v_{2}\right\rangle+\frac{1}{p}\left\|t v_{1}+(1-t) v_{2}\right\|^{p} \\
& \leq \frac{1}{q} \alpha_{k}\left\|z_{k}\right\|^{p}+\frac{1}{q}\left(1-\alpha_{k}\right)\left\|U^{k} z_{k}\right\|^{p}-\left(\alpha_{k}^{q}\left(1-\alpha_{k}\right)+\left(1-\alpha_{k}\right)^{q} \alpha_{k}\right) \\
& \times g\left(\left\|z_{k}-U^{k} z_{k}\right\|\right)-\alpha_{k}\left\langle J_{E_{1}}^{p}\left(z_{k}\right), t v_{1}+(1-t) v_{2}\right\rangle \\
& -\left(1-\alpha_{k}\right)\left\langle J_{E_{1}}^{p}\left(U^{k}\left(z_{k}\right)\right), t v_{1}+(1-t) v_{2}\right\rangle+\frac{1}{p}\left\|t v_{1}+(1-t) v_{2}\right\|^{p} \\
& =\alpha_{k}\left(\frac{1}{p}\left\|t v_{1}+(1-t) v_{2}\right\|^{p}-\left\langle J_{E_{1}}^{p}\left(z_{k}\right), t v_{1}+(1-t) v_{2}\right\rangle+\frac{1}{q}\left\|z_{k}\right\|^{p}\right) \\
& +\left(1-\alpha_{k}\right)\left(\frac{1}{p}\left\|t v_{1}+(1-t) v_{2}\right\|^{p}-\left\langle J_{E_{1}}^{p}\left(U^{k}\left(z_{k}\right)\right), t v_{1}+(1-t) v_{2}\right\rangle\right. \\
& \left.+\frac{1}{q}\left\|U^{k} z_{k}\right\|^{p}\right)-\left(\alpha_{k}^{q}\left(1-\alpha_{k}\right)+\left(1-\alpha_{k}\right)^{q} \alpha_{k}\right) g\left(\left\|z_{k}-U^{k} z_{k}\right\|\right) \\
& =\alpha_{k} \triangle_{p}\left(z_{k}, t v_{1}+(1-t) v_{2}\right)+\left(1-\alpha_{k}\right) \triangle_{p}\left(U^{k}\left(z_{k}\right), t v_{1}\right. \\
& \left.+(1-t) v_{2}\right)-\left(\alpha_{k}^{p}\left(1-\alpha_{k}\right)+\left(1-\alpha_{k}\right)^{p} \alpha_{k}\right) \times g\left(\left\|z_{k}-U^{k} z_{k}\right\|\right) \\
& \leq \alpha_{k} \triangle_{p}\left(z_{k}, t v_{1}+(1-t) v_{2}\right)+\left(1-\alpha_{k}\right)\left(k _ { k } \Delta _ { p } \left(z_{k}, t v_{1}\right.\right. \\
& \left.\left.+(1-t) v_{2}\right)+\Delta_{p}\left(z_{k}, t v_{1}+(1-t) v_{2}\right)+\mu_{k}\right)-\left(\alpha_{k}^{q}\left(1-\alpha_{k}\right)\right. \\
& \left.+\left(1-\alpha_{k}\right)^{q} \alpha_{k}\right) g\left(\left\|z_{k}-U^{k} z_{k}\right\|\right) \\
& \leq k_{k} \triangle_{p}\left(z_{k}, t v_{1}+(1-t) v_{2}\right)+\triangle_{p}\left(z_{k}, t v_{1}+(1-t) v_{2}\right)+\mu_{k} \\
& -\left(\alpha_{k}^{q}\left(1-\alpha_{k}\right)+\left(1-\alpha_{k}\right)^{q} \alpha_{k}\right) g\left(\left\|z_{k}-U^{k} z_{k}\right\|\right) \\
& \leq\left[k_{k}+1\right] \triangle_{p}\left(z_{k}, t v_{1}+(1-t) v_{2}\right)+\mu_{k} .
\end{aligned}
$$

From (27), (32) and (33), we have that $v \in C_{k+1}$. Hence, $C_{k+1}$ is convex.

Therefore $C_{n}$ is closed and convex for each $n \in \mathbb{N}$

Step two: We prove $\Gamma \subset C_{n}$ for any $n \geq 1$. Clearly $\Gamma \subset C_{1}$. Now assuming that $\Gamma \subset C_{n}$ for some $n \geq 1$. Let $v \in \Gamma$ then from (11) and Lemma 2.15, having considered $x_{n} \neq 0$, we get

$$
\begin{aligned}
\triangle_{p} & \left(z_{n}, v\right) \\
& =\frac{1}{q}\left\|J_{E_{1}}^{p} x_{n}-\lambda_{n} A^{*}\left(J_{E_{2}}^{p}\left(I-\Pi_{A C_{n}}^{p} T^{k}\right) A x_{n}\right)\right\|^{q}+\frac{1}{p}\|v\|^{p} \\
& -\left\langle J_{E_{1}}^{p} x_{n}-\lambda_{n} A^{*}\left(J_{E_{2}}^{p}\left(I-\Pi_{A C_{n}}^{p} T^{n}\right) A x_{n}\right), v\right\rangle \\
& =\frac{1}{q} \| J_{E_{1}}^{p} x_{n}-\lambda_{n} A^{*}\left(J_{E_{2}}^{p}\left(I-\Pi_{A C_{n}}^{p} T^{n}\right) A x_{n}\left\|^{q}+\frac{1}{p}\right\| v \|^{p}\right. \\
& -\left\langle J_{E_{1}}^{p} x_{n}, v\right\rangle+\left\langle\lambda_{n}\left(J_{E_{2}}^{p}\left(I-\Pi_{A C_{n}}^{p} T^{n}\right) A x_{n}, v\right\rangle .\right.
\end{aligned}
$$

Where

$$
\begin{aligned}
& \lambda_{n}\left\langle A^{*}\left(J_{E_{2}}^{p}\left(I-\Pi_{A C_{n}}^{p} T^{n}\right) A x_{n}, v\right\rangle\right. \\
& =\lambda_{n}\left\langle\left(J_{E_{2}}^{p}\left(I-\Pi_{A C_{n}}^{p} T^{n}\right) A x_{n}, A v+\Pi_{A C_{n}}^{p} T^{n} A x_{n}-\Pi_{A C_{n}}^{p} T^{n} A x_{n}\right\rangle\right. \\
& =\lambda_{n}\left\langle\left(J_{E_{2}}^{p}\left(I-\Pi_{A C_{n}}^{p} T^{n}\right) A x_{n}, A v-\Pi_{A C_{n}}^{p} T^{n} A x_{n}\right\rangle\right. \\
& +\lambda_{n}\left\langle\left(J_{E_{2}}^{p}\left(I-\Pi_{A C_{n}}^{p} T^{n}\right) A x_{n}, \Pi_{A C_{n}}^{p} T^{n} A x_{n}\right\rangle\right. \\
& =\lambda_{n}\left\langle\left(J_{E_{2}}^{p}\left(I-\Pi_{A C_{n}}^{p} T^{n}\right) A x_{n}, A v-\Pi_{A C_{n}}^{p} T^{n} A x_{n}\right\rangle\right. \\
& +\lambda_{n}\left\langle\left(J_{E_{2}}^{p}\left(I-\Pi_{A C_{n}}^{p} T^{n}\right) A x_{n}, \Pi_{A C_{n}}^{p} T^{n} A x_{n}-A x_{n}+A x_{n}\right\rangle\right. \\
& =\lambda_{n}\left\langle\left(J_{E_{2}}^{p}\left(I-\Pi_{A C_{n}}^{p} T^{n}\right) A x_{n}, A v-\Pi_{A C_{n}}^{p} T^{n} A x_{n}\right\rangle\right. \\
& -\lambda_{n}\left\langle\left(J_{E_{2}}^{p}\left(I-\Pi_{A C_{n}}^{p} T^{n}\right) A x_{n}, \Pi_{A C_{n}}^{p} T^{n} A x_{n}-A x_{n}\right\rangle\right. \\
& +\lambda_{n}\left\langle\left(J_{E_{2}}^{p}\left(I-\Pi_{A C_{n}}^{p} T^{n}\right) A x_{n}, A x_{n}\right\rangle\right.
\end{aligned}
$$

$$
\begin{aligned}
& =\lambda_{n}\left\langle\left(J_{E_{2}}^{p}\left(I-\Pi_{A C_{n}}^{p} T^{n}\right) A x_{n}, A v-A x_{n}+A x_{n}-\Pi_{A C_{n}}^{p} T^{n} A x_{n}\right\rangle\right. \\
& -\lambda_{n}\left\langle\left(J_{E_{2}}^{p}\left(I-\Pi_{A C_{n}}^{p} T^{n}\right) A x_{n}, A x_{n}-\Pi_{A C_{n}}^{p} T^{n} A x_{n}\right\rangle\right. \\
& +\lambda_{n}\left\langle\left(J_{E_{2}}^{p}\left(I-\Pi_{A C_{n}}^{p} T^{n}\right) A x_{n}, A x_{n}\right\rangle\right. \\
& =\lambda_{n}\left\langle\left(J_{E_{2}}^{p}\left(I-\Pi_{A C_{n}}^{p} T^{n}\right) A x_{n},\left(A v-A x_{n}\right)-\left(\Pi_{A C_{n}}^{p} T^{n} A x_{n}-A x_{n}\right)\right\rangle\right. \\
& -\lambda_{n}\left\langle\left(J_{E_{2}}^{p}\left(I-\Pi_{A C_{n}}^{p} T^{n}\right) A x_{n}, A x_{n}-\Pi_{A C_{n}}^{p} T^{n} A x_{n}\right\rangle\right. \\
& +\lambda_{n}\left\langle\left(J_{E_{2}}^{p}\left(I-\Pi_{A C_{n}}^{p} T^{n}\right) A x_{n}, A x_{n}\right\rangle\right. \\
& =-\lambda_{n}\left\langle\left(J_{E_{2}}^{p}\left(\Pi_{A C_{n}}^{p} T^{n}-I\right) A x_{n},\left(A v-A x_{n}\right)-\left(\Pi_{A C_{n}}^{p} T^{n} A x_{n}-A x_{n}\right)\right\rangle\right. \\
& -\lambda_{n}\left\langle\left(J_{E_{2}}^{p}\left(I-\Pi_{A C_{n}}^{p} T^{n}\right) A x_{n}, A x_{n}-\Pi_{A C_{n}}^{p} T^{n} A x_{n}\right\rangle\right. \\
& +\lambda_{n}\left\langle\left(J_{E_{2}}^{p}\left(I-\Pi_{A C_{n}}^{p} T^{n}\right) A x_{n}, A x_{n}\right\rangle .\right.
\end{aligned}
$$

By the assumption that $A\left(C_{n}\right)$ is closed and convex and Lemma 2.5 and by the variational inequality for the Bregman projection of zero onto $A\left(C_{n}\right)-A x_{n}$, as in Definition 2.6, we arive at

$$
\left\langle\left(J_{E_{2}}^{p}\left(\Pi_{A C_{n}}^{p} T^{n}-I\right) A x_{n},\left(A v-A x_{n}\right)-\left(\Pi_{A C_{n}}^{p} T^{n} A x_{n}-A x_{n}\right)\right\rangle \geq 0\right.
$$

and therefore,

$$
\begin{aligned}
\lambda_{n} & \left\langle J_{E_{2}}^{p}\left(I-\Pi_{A C_{n}}^{p} T^{n}\right) A x_{n}, A v\right\rangle \\
& \leq \lambda_{n}\left\langle J_{E_{2}}^{p}\left(I-\Pi_{A C_{n}}^{p} T^{n}\right) A x_{n}, A x_{n}\right\rangle \\
& -\lambda_{n}\left\langle J_{E_{2}}^{p}\left(I-\Pi_{A C_{n}}^{p} T^{n}\right) A x_{n}, A x_{n}-\Pi_{A C_{n}}^{p} T^{n} A x_{n}\right\rangle .
\end{aligned}
$$

In addition, from Lemma 2.15, we have that

$$
\begin{aligned}
\frac{1}{q} \| J_{E_{1}}^{p} x_{n}-\lambda_{n} A^{*}\left(J_{E_{2}}^{p}\left(I-\Pi_{A C_{n}}^{p} T^{n}\right) A x_{n} \|^{q}\right. \\
\quad \leq \frac{1}{q}\left\|J_{E_{1}}^{p} x_{n}\right\|^{q}-\lambda_{n}\left\langle x_{n}, A^{*} J_{E_{2}}^{p}\left(I-\Pi_{A C_{n}}^{p} T^{n}\right) A x_{n}\right\rangle \\
\quad+\frac{1}{q} \bar{\sigma}_{q}\left(J_{E_{1}}^{p} x_{n}, \lambda_{n} A^{*} J_{E_{2}}^{p}\left(I-\Pi_{A C_{n}}^{p} T^{n}\right) A x_{n}\right) \\
\quad=\frac{1}{q}\left\|J_{E_{1}}^{p} x_{n}\right\|^{q}-\lambda_{n}\left\langle A x_{n}, J_{E_{2}}^{p}\left(I-\Pi_{A C_{n}}^{p} T^{n}\right) A x_{n}\right\rangle \\
\quad+\frac{1}{q} \bar{\sigma}_{q}\left(J_{E_{1}}^{p} x_{n}, \lambda_{n} A^{*} J_{E_{2}}^{p}\left(I-\Pi_{A C_{n}}^{p} T^{n}\right) A x_{n}\right) \\
\quad=\frac{1}{q}\left\|x_{n}\right\|^{p}-\lambda_{n}\left\langle A x_{n}, J_{E_{2}}^{p}\left(I-\Pi_{A C_{n}}^{p} T^{n}\right) A x_{n}\right\rangle \\
\quad+\frac{1}{q} \bar{\sigma}_{q}\left(J_{E_{1}}^{p} x_{n}, \lambda_{n} A^{*} J_{E_{2}}^{p}\left(I-\Pi_{A C_{n}}^{p} T^{n}\right) A x_{n}\right)
\end{aligned}
$$

and

$$
\begin{aligned}
& \frac{1}{q} \bar{\sigma}_{q}\left(J_{E_{1}}^{p} x_{n}, \lambda_{n} A^{*} J_{E_{2}}^{p}\left(I-\Pi_{A C_{n}}^{p} T^{n}\right) A x_{n}\right) \\
& \quad=G_{q} \int_{0}^{1} \frac{\left(\left\|J_{E_{1}}^{p} x_{n}-t \lambda_{n} A^{*} J_{E_{2}}^{p}\left(I-\Pi_{A C_{n}}^{p} T^{n}\right) A x_{n}\right\| \vee\left\|J_{E_{1}}^{p} x_{n}\right\|\right)^{q}}{t} \times \\
& \rho_{E^{*}}\left(\frac{t\left\|\lambda_{n} A^{*} J_{E_{2}}^{p}\left(I-\Pi_{A C_{n}}^{p} T^{n}\right) A x_{n}\right\|}{\left(\left\|J_{E_{1}}^{p} x_{n}-t \lambda_{n} A^{*} J_{E_{2}}^{p}\left(I-\Pi_{A C_{n}}^{p} T^{n}\right) A x_{n}\right\| \vee\left\|J_{E_{1}}^{p} x_{n}\right\|\right)}\right) d t,
\end{aligned}
$$

for every $t \in[0,1]$. 
But

$$
\begin{aligned}
& \left\|J_{E_{1}}^{p} x_{n}-t \lambda_{n} A^{*} J_{E_{2}}^{p}\left(I-\Pi_{A C_{n}}^{p} T^{n}\right) A x_{n}\right\| \\
& \leq\left\|x_{n}\right\|^{p-1}+\left\|\lambda_{n} A^{*} J_{E_{2}}^{p}\left(I-\Pi_{A C_{n}}^{p} T^{n}\right) A x_{n}\right\| .
\end{aligned}
$$

From (12), suppose that

$$
\lambda_{n}=\frac{\tau_{n}}{\|A\|} \frac{\left\|x_{n}\right\|^{p-1}}{\left\|J_{E_{2}}^{p}\left(I-\Pi_{A C_{n}}^{p} T^{n}\right) A x_{n}\right\|}
$$

then we have

$$
\left\|J_{E_{1}}^{p} x_{n}-t \lambda_{n} A^{*} J_{E_{2}}^{p}\left(I-\Pi_{A C_{n}}^{p} T^{n}\right) A x_{n}\right\| \leq 2\left\|x_{n}\right\|^{p-1}
$$

and that

$\left\|x_{n}\right\|^{p-1} \leq\left\|J_{E_{1}}^{p} x_{n}-t \lambda_{n} A^{*} J_{E_{2}}^{p}\left(I-\Pi_{A C_{n}}^{p} T^{n}\right) A x_{n}\right\| \vee\left\|J_{E_{1}}^{p} x_{n}\right\| \leq 2\left\|x_{n}\right\|^{p-1}$.

$$
\begin{aligned}
& =\triangle_{p}\left(x_{n}, v\right)+2^{q} G_{q}\left\|x_{n}\right\|^{p} \rho_{E_{1}^{*}}\left(\tau_{n}\right) \\
& \left.-\lambda_{n}\left\langle\left(J_{E_{2}}^{p}\left(I-\Pi_{A C_{n}}^{p} T^{n}\right) A x_{n}\right), A x_{n}-\Pi_{A C_{n}}^{p} T^{n}\right) A x_{n}\right\rangle .
\end{aligned}
$$

Substituting (13) and (12) into (42), we have that

$$
\begin{aligned}
& \triangle_{p}\left(z_{n}, v\right) \\
& \quad \leq \triangle_{p}\left(x_{n}, v\right)+\frac{\left.\gamma\left\langle J_{E_{2}}^{p}\left(I-\Pi_{A C_{n}}^{p} T^{n}\right) A x_{n}, A x_{n}-\Pi_{A C_{n}}^{p} T^{n}\right) A x_{n}\right\rangle}{\left.\|A\| \| J_{E_{2}}^{p}\left(I-\Pi_{A C_{n}}^{p} T^{n}\right) A x_{n}\right) \|} \\
& \quad-\frac{\left.\left\langle J_{E_{2}}^{p}\left(I-\Pi_{A C_{n}}^{p} T^{n}\right) A x_{n}, A x_{n}-\Pi_{A C_{n}}^{p} T^{n}\right) A x_{n}\right\rangle}{\left\|A^{*}\right\|\left\|J_{E_{2}}^{p}\left(I-\Pi_{A C_{n}}^{p} T^{n}\right) A x_{n}\right\|} \\
& \quad=\triangle_{p}\left(x_{n}, v\right)-[1-\gamma] \\
& \times \frac{\left.\left\langle J_{E_{2}}^{p}\left(I-\Pi_{A C_{n}}^{p} T^{n}\right) A x_{n}, A x_{n}-\Pi_{A C_{n}}^{p} T^{n}\right) A x_{n}\right\rangle}{\left\|A^{*}\right\|\left\|J_{E_{2}}^{p}\left(I-\Pi_{A C_{n}}^{p} T^{n}\right) A x_{n}\right\|}
\end{aligned}
$$

From Definition 2.2(2), we have that from Eqs. (38) and (13)

$$
\begin{aligned}
& \rho_{E_{1}^{*}}\left(\frac{t\left\|\lambda_{n} A^{*} J_{E_{2}}^{p}\left(I-\Pi_{A C_{n}}^{p} T^{n}\right) A x_{n}\right\|}{\left(\left\|J_{E_{1}}^{p} x_{n}-t \lambda_{n} A^{*} J_{E_{2}}^{p}\left(I-\Pi_{A C_{n}}^{p} T^{n}\right) A x_{n}\right\| \vee\left\|J_{E_{1}}^{p} x_{n}\right\|\right)}\right) \\
& \quad \leq \rho_{E_{1}^{*}}\left(\frac{t\left\|\lambda_{n} A^{*} J_{E_{2}}^{p}\left(I-\Pi_{A C_{n}}^{p} T^{n}\right) A x_{n}\right\|}{\left\|x_{n}\right\|^{p-1}}\right) \\
& \quad=\rho_{E_{1}^{*}}\left(t \tau_{n}\right) .
\end{aligned}
$$

Substituting Eqs. (38) and (39) into (37), and since $t \leq \tau_{n}$ and $\rho_{E_{1}^{*}}$ is nondecreasing then, we have that

$$
\begin{gathered}
\frac{1}{q} \bar{\sigma}_{q}\left(J_{E_{2}}^{p} x_{n}, \lambda_{n} A^{*} J_{E_{2}}^{p}\left(I-\Pi_{A C_{n}}^{p} T^{n}\right) A x_{n}\right) \\
\leq 2^{q} G_{q}\left\|x_{n}\right\|^{(p-1) q} \int_{0}^{\tau} \frac{\rho_{E_{1}^{*}}(t)}{t} d t \\
\leq 2^{q} G_{q}\left\|x_{n}\right\|^{p} \int_{0}^{\tau} \frac{\rho_{E_{1}^{*}}\left(\tau_{n}\right)}{\tau_{n}} d t \\
\leq 2^{q} G_{q}\left\|x_{n}\right\|^{p} \rho_{E_{1}^{*}}\left(\tau_{n}\right) .
\end{gathered}
$$

Substituting Eq. (40) into (36), we have that

$$
\begin{aligned}
& \frac{1}{q}\left\|J_{E_{1}}^{p} x_{n}-\lambda_{n} A^{*} J_{E_{2}}^{p}\left(I-\Pi_{A C_{n}}^{p} T^{n}\right) A x_{n}\right\|^{q} \\
& \quad \leq \frac{1}{q}\left\|x_{n}\right\|^{p}-\lambda_{n}\left\langle A x_{n}, J_{E_{2}}^{p}\left(I-\Pi_{A C_{n}}^{p} T^{n}\right) A x_{n}\right\rangle+2^{q} G_{q}\left\|x_{n}\right\|^{p} \rho_{E_{1}^{*}}\left(\tau_{n}\right) \\
& \quad=\frac{1}{q}\left\|J_{E_{1}}^{p} x_{n}\right\|^{q}-\lambda_{n}\left\langle\left(J_{E_{2}}^{p}\left(I-\Pi_{A C_{n}}^{p} T^{n}\right) A x_{n}, A x_{n}\right\rangle\right. \\
& \quad+2^{q} G_{q}\left\|x_{n}\right\|^{p} \rho_{E_{1}^{*}}\left(\tau_{n}\right) .
\end{aligned}
$$

Substituting Eqs. (35) and (41) into (34), we have that

$$
\begin{aligned}
\triangle_{p} & \left(z_{n}, v\right) \leq \frac{1}{q}\left\|x_{n}\right\|^{p}+\frac{1}{p}\|v\|^{p} \\
& -\left\langle J_{E_{1}}^{p} x_{n}, v\right\rangle+2^{q} G_{q}\left\|x_{n}\right\|^{p} \rho_{E_{1}^{*}}\left(\tau_{n}\right) \\
& \left.-\lambda_{n}\left\langle\left(J_{E_{2}}^{p}\left(I-\Pi_{A C_{n}}^{p} T^{n}\right) A x_{n}\right), A x_{n}-\Pi_{A C_{n}}^{p} T^{n}\right) A x_{n}\right\rangle
\end{aligned}
$$

$$
\triangle_{p}\left(z_{n}, v\right) \leq \triangle_{p}\left(x_{n}, v\right) .
$$

For $x_{n}=0$ we have

$$
\triangle_{p}\left(x_{n}, v\right)=\frac{1}{p}\|v\|^{p}
$$

and from (45), we have

$$
\begin{aligned}
& \triangle_{p}\left(z_{n}, v\right)=\frac{1}{q}\left\|\lambda_{n} A^{*} J_{E_{2}}^{p}\left(I-\Pi_{A C_{n}}^{p} T^{n}\right) A x_{n}\right\|^{q}+\triangle_{p}\left(x_{n}, v\right) \\
& \left.\quad+\lambda_{n}\left\langle J_{E_{2}}^{p}\left(I-\Pi_{A C_{n}}^{p} T^{n}\right) A x_{n}\right), A v\right\rangle .
\end{aligned}
$$

Substituting Eq. (35) into Eq. (46), we have that

$$
\begin{aligned}
& \triangle_{p}\left(z_{n}, v\right) \leq \frac{1}{q}\left\|\lambda_{n} A^{*} J_{E_{2}}^{p}\left(I-\Pi_{A C_{n}}^{p} T^{n}\right) A x_{n}\right\|^{q} \\
& \quad+\triangle_{p}\left(x_{n}, v\right)+\lambda_{n}\left\langle J_{E_{2}}^{p}\left(I-\Pi_{A C_{n}}^{p} T^{n}\right) A x_{n}, A x_{n}\right\rangle \\
& \left.\quad-\lambda_{n}\left\langle J_{E_{2}}^{p}\left(I-\Pi_{A C_{n}}^{p} T^{n}\right) A x_{n}, A x_{n}-\Pi_{A C_{n}}^{p} T^{n}\right) A x_{n}\right\rangle
\end{aligned}
$$

But, from (13), we have that

$$
\begin{aligned}
& \frac{1}{q}\left\|\lambda_{n} A^{*} J_{E_{2}}^{p}\left(I-\Pi_{A C_{n}}^{p} T^{n}\right) A x_{n}\right\|^{q} \\
& \quad=\frac{1}{q} \frac{1}{\|A\|^{p}} \frac{\left.\left\langle J_{E_{2}}^{p}\left(I-\Pi_{A C_{n}}^{p} T^{n}\right) A x_{n}, A x_{n}-\Pi_{A C_{n}}^{p} T^{n}\right) A x_{n}\right\rangle^{p}}{\| J_{E_{2}}^{p}\left(I-\Pi_{A C_{n}}^{p} T^{n} A x_{n} \|^{p}\right.} .
\end{aligned}
$$

Substituting (48) into (47), we have that

$$
\begin{aligned}
& \triangle_{p}\left(z_{n}, v\right) \\
& \quad \leq \frac{1}{q} \frac{1}{\|A\|^{p}} \frac{\left\langle J_{E_{2}}^{p}\left(I-\Pi_{A C_{n}}^{p} T^{n}\right) A x_{n}, A x_{n}-\Pi_{A C_{n}}^{p} T^{n} A x_{n}\right\rangle^{p}}{\left\|J_{E_{2}}^{p}\left(I-\Pi_{A C_{n}}^{p} T^{n}\right) A x_{n}\right\|^{p}} \\
& +\triangle_{p}\left(x_{n}, v\right)+\lambda_{n}\left\langle J_{E_{2}}^{p}\left(I-\Pi_{A C_{n}}^{p} T^{n}\right) A x_{n}, A x_{n}\right\rangle \\
& -\lambda_{n}\left\langle J_{E_{2}}^{p}\left(I-\Pi_{A C_{n}}^{p} T^{n}\right) A x_{n}, A x_{n}-\Pi_{A C_{n}}^{p} T^{n} A x_{n}\right\rangle \\
& \leq\left(1-\frac{1}{p}\right) \frac{1}{\|A\|^{p}} \frac{\left\langle J_{E_{2}}^{p}\left(I-\Pi_{A C_{n}}^{p} T^{n}\right) A x_{n}, A x_{n}-\Pi_{A C_{n}}^{p} T^{n} A x_{n}\right\rangle^{p}}{\left\|J_{E_{2}}^{p}\left(I-\Pi_{A C_{n}}^{p} T^{n}\right) A x_{n}\right\|^{p}}
\end{aligned}
$$




$$
\begin{aligned}
& +\triangle_{p}\left(x_{n}, v\right)+\lambda_{n}\left\langle A^{*} J_{E_{2}}^{p}\left(I-\Pi_{A C_{n}}^{p} T^{n}\right) A x_{n}, x_{n}\right\rangle \\
& -\frac{1}{\|A\|^{p}} \frac{\left\langle J_{E_{2}}^{p}\left(I-\Pi_{A C_{k}}^{p} T^{n}\right) A x_{n}, A x_{n}-\Pi_{A C_{n}}^{p} T^{n} A x_{n}\right\rangle^{p}}{\left\|J_{E_{2}}^{p}\left(I-\Pi_{A C_{n}}^{p} T^{n}\right) A x_{n}\right\|^{p}} \\
& \times\left\langle J_{E_{2}}^{p}\left(I-\Pi_{A C_{n}}^{p} T^{n}\right) A x_{n}, A x_{n}-\Pi_{A C_{n}}^{p} T^{n} A x_{n}\right\rangle \\
& \leq\left(1-\frac{1}{p}\right) \frac{1}{\|A\|^{p}} \frac{\left\langle J_{E_{2}}^{p}\left(I-\Pi_{A C_{n}}^{p} T^{n}\right) A x_{n}, A x_{n}-\Pi_{A C_{n}}^{p} T^{n} A x_{n}\right\rangle^{p}}{\left\|J_{E_{2}}^{p}\left(I-\Pi_{A C_{n}}^{p} T^{n}\right) A x_{n}\right\|^{p}} \\
& +\triangle_{p}\left(x_{n}, v\right)+\lambda_{n}\left\|A^{*} J_{E_{2}}^{p}\left(I-\Pi_{A C_{n}}^{p} T^{n}\right) A x_{n}\right\|\left\|x_{n}\right\| \\
& -\frac{1}{\|A\|^{p}} \frac{\left\langle J_{E_{2}}^{p}\left(I-\Pi_{A C_{n}}^{p} T^{n}\right) A x_{n}, A x_{n}-\Pi_{A C_{n}}^{p} T^{n} A x_{n}\right\rangle^{p}}{\left\|J_{E_{2}}^{p}\left(I-\Pi_{A C_{n}}^{p} T^{n}\right) A x_{n}\right\|^{p}} \\
& \times\left\langle J_{E_{2}}^{p}\left(I-\Pi_{A C_{n}}^{p} T^{n}\right) A x_{n}, A x_{n}-\Pi_{A C_{n}}^{p} T^{n} A x_{n}\right\rangle \\
& =\triangle_{p}\left(x_{n}, v\right) \\
& \left.-\frac{1}{p}\right) \frac{1}{\|A\|^{p}} \frac{\left\langle J_{E_{2}}^{p}\left(I-\Pi_{A C_{n}}^{p} T^{n}\right) A x_{n}, A x_{n}-\Pi_{A C_{n}}^{p} T^{n} A x_{n}\right\rangle^{p}}{\left\|J_{E_{2}}^{p}\left(I-\Pi_{A C_{n}}^{p} T^{n}\right) A x_{n}\right\|^{p}} \\
& \left.=\triangle_{p}\left(x_{n}, v\right)-\frac{1}{p}\right) \frac{1}{\|A\|^{p}} \\
& \times \frac{\left.\left\langle J_{E_{2}}^{p}\left(I-\Pi_{A C_{n}}^{p} T^{n}\right) A x_{n}, A x_{n}-\Pi_{A C_{n}}^{p} T^{n}\right) A x_{n}\right\rangle^{p}}{\left\|J_{E_{2}}^{p}\left(I-\Pi_{A C_{n}}^{p} T^{n}\right) A x_{n}\right\|^{p}}
\end{aligned}
$$

This implies that

$$
\triangle_{p}\left(z_{n}, v\right) \leq \triangle_{p}\left(x_{n}, v\right) .
$$

In addition, it follows from (11), Definition 2.4, Lemma 2.16 and $p \in(1, \infty)$ that

$$
\begin{aligned}
\triangle_{p} & \left(y_{n}, v\right) \\
& =\Delta_{p}\left(J_{E_{1}^{*}}^{q}\left(\alpha_{n} J_{E_{1}}^{p}\left(z_{n}\right)+\left(1-\alpha_{n}\right) J_{E_{1}}^{p}\left(U^{n}\left(z_{n}\right)\right)\right), v\right) \\
& =\frac{1}{q}\left\|\alpha_{n} J_{E_{1}}^{p} z_{n}+\left(1-\alpha_{n}\right) J_{E_{1}}^{p} U_{n}^{z}\right\|^{q}-\left\langle\alpha_{n} J_{E_{1}}^{p} z_{n}\right. \\
& \left.+\left(1-\alpha_{n}\right) J_{E_{1}}^{p} U^{n} z_{n}, v\right\rangle+\frac{1}{p}\|v\|^{p} \\
& =\frac{1}{q}\left\|\alpha_{n} J_{E_{1}}^{p} z_{n}+\left(1-\alpha_{n}\right) J_{E_{1}}^{p} U^{n} z_{n}\right\|^{p}-\alpha_{n}\left\langle J_{E_{1}}^{p} z_{n}, v\right\rangle \\
& -\left(1-\alpha_{n}\right)\left\langle J_{E_{1}}^{p}\left(U^{n}\left(z_{n}\right)\right), v\right\rangle+\frac{1}{p}\|v\|^{p} \\
& \leq \frac{1}{q} \alpha_{n}\left\|z_{n}\right\|^{p}+\frac{1}{q}\left(1-\alpha_{n}\right)\left\|U^{n} z_{n}\right\|^{p}-\left(\alpha_{n}^{q}\left(1-\alpha_{n}\right)+\left(1-\alpha_{n}\right)^{q} \alpha_{n}\right) \\
& \times g\left(\left\|z_{n}-U^{n} z_{n}\right\|\right)-\alpha_{n}\left\langle J_{E_{1}}^{p}\left(z_{n}\right), v\right\rangle-\left(1-\alpha_{n}\right)\left\langle J_{E_{1}}^{p}\left(U^{n}\left(z_{n}\right)\right), v\right\rangle \\
& +\frac{1}{p}\|v\|^{p} \\
& =\alpha_{n}\left(\frac{1}{p}\|v\|^{p}-\left\langle J_{E_{1}}^{p}\left(z_{n}\right), v\right\rangle+\frac{1}{q}\left\|z_{n}\right\|^{p}\right)+\left(1-\alpha_{n}\right)\left(\frac{1}{p}\|v\|^{p}\right. \\
& \left.-\left\langle J_{E_{1}}^{p}\left(U^{n}\left(z_{n}\right)\right), v\right\rangle+\frac{1}{q}\left\|U^{n} z_{n}\right\|^{p}\right)-\left(\alpha_{n}^{q}\left(1-\alpha_{n}\right)\right. \\
& \left.+\left(1-\alpha_{n}\right)^{q} \alpha_{n}\right) g\left(\left\|z_{n}-U^{n} z_{n}\right\|\right) \\
& =\alpha_{n} \triangle_{p}\left(z_{n}, v\right)+\left(1-\alpha_{n}\right) \triangle_{p}\left(U^{n}\left(z_{n}\right), v\right) \\
& -\left(\alpha_{n}^{p}\left(1-\alpha_{n}\right)+\left(1-\alpha_{n}\right)^{p} \alpha_{n}\right) \times g\left(\left\|z_{n}-U^{n} z_{n}\right\|\right) \\
& \leq \alpha_{n} \triangle_{p}\left(z_{n}, v\right)+\left(1-\alpha_{n}\right)\left(k_{n} \triangle_{p}\left(z_{n}, v\right)+\Delta_{p}\left(z_{n}, v\right)+\mu_{n}\right)
\end{aligned}
$$

From Eqs. (44), (51) and (53), we have that $v \in C_{n}$ and $\Gamma \subset$ $C_{n}$ for any $n \geq 1$. Step three: we show that $\left\{x_{n}\right\}$ is Cauchy sequence. For $v \in C_{n}$, and $x_{n+1}=\Pi_{C_{n+1}}^{p}\left(x_{1}\right) \in C_{n+1} \subset C_{n}$, then from Definition 2.6, we have that

$$
\begin{aligned}
0 & \leq\left\langle J_{E_{1}}^{p} \Pi_{C_{n+1}}^{p}\left(x_{1}\right)-J_{E_{1}}^{p}\left(x_{1}\right), v-\Pi_{C_{n+1}}^{p}\left(x_{1}\right)\right\rangle \\
& =\left\langle J_{E_{1}}^{p} \Pi_{C_{n+1}}^{p}\left(x_{1}\right), v\right\rangle-\left\langle J_{E_{1}}^{p}\left(x_{1}\right), v\right\rangle \\
& -\left\langle J_{E_{1}}^{p} \Pi_{C_{n+1}}^{p}\left(x_{1}\right), \Pi_{C_{n+1}}^{p}\left(x_{1}\right)\right\rangle+\left\langle J_{E_{1}}^{p}\left(x_{1}\right), \Pi_{C_{n+1}}^{p}\left(x_{1}\right)\right\rangle .
\end{aligned}
$$

This implies that

$$
\begin{gathered}
\left|\left\langle J_{E_{1}}^{p} \Pi_{C_{n+1}}^{p}\left(x_{1}\right), \Pi_{C_{n+1}}^{p}\left(x_{1}\right)\right\rangle\right| \leq \mid\left\langle J_{E_{1}}^{p} \Pi_{C_{n+1}}^{p}\left(x_{1}\right), v\right\rangle \\
-\left\langle J_{E_{1}}^{p}\left(x_{1}\right), v\right\rangle+\left\langle J_{E_{1}}^{p}\left(x_{1}\right), \Pi_{C_{n+1}}^{p}\left(x_{1}\right)\right\rangle \mid .
\end{gathered}
$$

That is

$\left\|\Pi_{C_{n+1}}^{p}\left(x_{1}\right)\right\|^{p} \leq\left\|\Pi_{C_{n+1}}^{p}\left(x_{1}\right)\right\|^{p-1}\|v\|+\left\|x_{1}\right\|^{p-1}\|v\|+\left\|x_{1}\right\|^{p-1}\left\|\Pi_{C_{n+1}}^{p}\left(x_{1}\right)\right\|$.

Observe that if

$$
\begin{aligned}
\left\|\Pi_{C_{n+1}}^{p}\left(x_{1}\right)\right\|^{p-1} & <2\left\|x_{1}\right\|^{p-1} \\
\text { then we have }\left\|\Pi_{C_{n+1}}^{p}\left(x_{1}\right)\right\| & <2^{q-1}\left\|x_{1}\right\|
\end{aligned}
$$

and so we are done. Otherwise, let

$$
t_{x_{1}}=\left(\frac{\left\|\Pi_{C_{n+1}}^{p}\left(x_{1}\right)\right\|}{\left\|x_{1}\right\|}\right)^{p-1} \geq 2 .
$$

For $x_{1} \neq 0$ and using (54) we have that

$$
\begin{gathered}
\left\|\Pi_{C_{n+1}}^{p}\left(x_{1}\right)\right\|\left(\left\|\Pi_{C_{n+1}}^{p}\left(x_{1}\right)\right\|^{p-1}-\left\|x_{1}\right\|^{p-1}\right) \\
\leq\|v\|\left(\left\|\Pi_{C_{n+1}}^{p}\left(x_{1}\right)\right\|^{p-1}+\left\|x_{1}\right\|^{p-1}\right)
\end{gathered}
$$

and so

$$
\left\|\Pi_{C_{n+1}}^{p}\left(x_{1}\right)\right\| \leq\|v\| \frac{\left\|\Pi_{C_{n+1}}^{p}\left(x_{1}\right)\right\|^{p-1}+\left\|x_{1}\right\|^{p-1}}{\left\|\Pi_{C_{n+1}}^{p}\left(x_{1}\right)\right\|^{p-1}-\left\|x_{1}\right\|^{p-1}} .
$$

From (56), since the function $h\left(t_{x_{1}}\right)=\frac{t_{x_{1}}+1}{t_{x_{1}}-1}$ is decreasing for $t_{x_{1}}>1$ we arive at

$$
\left\|\Pi_{C_{n+1}}^{p}\left(x_{1}\right)\right\| \leq \min _{v \in C_{n+1}}\|v\| h\left(t_{x_{1}}\right) \leq \min _{v \in C_{n+1}}\|v\| h(2)=3 \min _{v \in C_{n+1}}\|v\| .
$$

If $x_{1}=0$ then from (54), we have that

$$
\left\|\Pi_{C_{n+1}}^{p}\left(x_{1}\right)\right\| \leq \min _{v \in C_{n+1}}\|v\| .
$$

From (55), (57) and (58), we have that $x_{n+1}=\Pi_{C_{n+1}}^{p}\left(x_{1}\right)$ is 
bounded.

For any $n \geq 1$, by Definition 2.6, we have that

$$
\begin{aligned}
& 0 \leq \triangle_{p}\left(J_{E_{1}}^{p} \Pi_{C_{n}}^{p}\left(x_{1}\right), \Pi_{C_{n+1}}^{p}\left(x_{1}\right)\right) \\
& \leq \triangle_{p}\left(x_{1}, \Pi_{C_{n+1}}^{p}\left(x_{1}\right)\right)-\Delta_{p}\left(x_{1}, \Pi_{C_{n}}^{p}\left(x_{1}\right)\right)
\end{aligned}
$$

From (59), we have that

$$
\triangle_{p}\left(x_{1}, \Pi_{C_{n}}^{p}\left(x_{1}\right)\right) \leq \triangle_{p}\left(x_{1}, \Pi_{C_{n+1}}^{p}\left(x_{1}\right)\right) \text {. }
$$

Thus $\left\{\triangle_{p}\left(x_{1}, \Pi_{C_{n}}^{p}\left(x_{1}\right)\right)\right\}$ is nondecreasing. Therefore by boundedness of $\Pi_{C_{n}}^{p}\left(x_{1}\right)$, we have that $\lim _{n \rightarrow \infty} \Delta_{p}\left(x_{1}, \Pi_{C_{n}}^{p}\left(x_{1}\right)\right)$ exists. Let $m, n \in N m<n$. From $x_{n}=\Pi_{C_{n}}^{p}\left(x_{1}\right) \subset C_{n}$ and (59), we have that

$$
\triangle_{p}\left(\Pi_{C_{n}}^{p}\left(x_{1}\right), \Pi_{C_{m}}^{p}\left(x_{1}\right)\right) \leq \Delta_{p}\left(x_{1}, \Pi_{C_{m}}^{p}\left(x_{1}\right)\right)-\Delta_{p}\left(x_{1}, \Pi_{C_{n}}^{p}\left(x_{1}\right)\right) .
$$

Since $\lim _{n \rightarrow \infty} \triangle_{p}\left(x_{1}, \Pi_{C_{n}}^{p}\left(x_{1}\right)\right)$ exists, it follows from (60) that

$$
\triangle_{p}\left(\Pi_{C_{m}}^{p}\left(x_{1}\right), \Pi_{C_{n}}^{p}\left(x_{1}\right)\right) \rightarrow 0 \text { as } m, n \rightarrow \infty .
$$

Therefore $\left\{\Pi_{C_{n}}^{p}\left(x_{1}\right)\right\}$ is a Cauchy sequence.

Step four: we will show that $\lim _{n \rightarrow \infty} \triangle_{p}\left(z_{n}, U z_{n}\right)=0$ and $\lim _{n \rightarrow \infty} \triangle_{p}$ $\left(A x_{n}, \Pi_{A C_{n}}^{p} T^{n} A x_{n}\right)=0$. Let $x_{n+1}=\Pi_{C_{n+1}}^{p}\left(x_{1}\right) \subset C_{n+1} \subset C_{n}$.

From 11, we have that

$$
\triangle_{p}\left(z_{n}, v\right) \leq \triangle_{p}\left(x_{n}, v\right) \Leftrightarrow \frac{1}{p}\left\|z_{n}-v\right\|^{p} \leq \frac{1}{p}\left\|x_{n}-v\right\|^{p} .
$$

Therefore $\left\{z_{n}\right\}$ is bounded as $\left\{x_{n}\right\}$ is bounded.

Hence, from Lemma 2.9 and 2.10, we have

$$
\begin{aligned}
\triangle_{p}\left(z_{n}, x_{n}\right)= & \Delta_{p}\left(z_{n}, x_{n+1}\right)+\triangle_{p}\left(x_{n+1}, x_{n}\right) \\
& -\left\langle z_{n}-x_{n+1}, J^{p} x_{n}-J^{p} x_{n+1}\right\rangle \\
& \leq \triangle_{p}\left(x_{n}, x_{n+1}\right)+\triangle_{p}\left(x_{n+1}, x_{n}\right) \\
& -\left\langle z_{n}-x_{n+1}, J^{p} x_{n}-J^{p} x_{n+1}\right\rangle \\
& =\left\langle J^{p} x_{n+1}-J^{p} x_{n}, x_{n+1}-x_{n},\right\rangle \\
& -\left\langle z_{n}-x_{n+1}, J^{p} x_{n}-J^{p} x_{n+1}\right\rangle \\
& \rightarrow 0 \text { as } n \rightarrow \infty .
\end{aligned}
$$

Now, for $x_{n} \neq 0$ for all $n \in N$, from (43), we have

$$
\triangle_{p}\left(z_{n}, v\right) \leq \triangle_{p}\left(x_{n}, v\right)-[1-\gamma]\left\|A x_{n}-\Pi_{A C_{n}}^{p} T^{n} A x_{n}\right\|^{p-1} .
$$

This implies that

$$
\begin{aligned}
& \left\|A x_{n}-\Pi_{A C_{n}}^{p} T^{n} A x_{n}\right\| \leq\left(\frac{\triangle_{p}\left(x_{n}, v\right)-\triangle_{p}\left(z_{n}, v\right)}{[1-\gamma]}\right)^{\frac{1}{p-1}} \\
& =\left(\frac{\frac{1}{q}\left(\left\|z_{n}\right\|^{p}-\left\|x_{n}\right\|^{p}\right)+\left\langle J_{E_{1}}^{p} z_{n}-J_{E_{1}}^{p} x_{n}, v\right\rangle}{[1-\gamma]}\right)^{\frac{1}{p-1}}
\end{aligned}
$$

and therefore we have that

$$
\lim _{n \rightarrow \infty}\left\|A x_{n}-\Pi_{A C_{n}}^{p} T^{n} A x_{n}\right\|=0 .
$$

For $x_{n}=0$ for all $n \in N$, from (49), we have

$$
\begin{aligned}
& \triangle_{p}\left(z_{n}, v\right) \leq \triangle_{p}\left(x_{n}, v\right) \\
& \quad-\frac{1}{p} \frac{\left\langle J_{E_{2}}^{p}\left(I-\Pi_{A C_{n}}^{p} T^{n}\right) A x_{n}, A x_{n}-\Pi_{A C_{n}}^{p} T^{n} A x_{n}\right\rangle^{p}}{\left.\|A\|^{p} \| J_{E_{2}}^{p}\left(I-\Pi_{A C_{n}}^{p} T^{n}\right) A x_{n}\right) \|^{p}} .
\end{aligned}
$$

This implies that

$$
\begin{aligned}
\| A x_{n} & -\Pi_{A C_{n}}^{p} T^{n} A x_{n} \| \leq \frac{p^{\frac{1}{p^{2}-p}}}{\|A\|^{p}}\left(\triangle_{p}\left(x_{n}, v\right)-\triangle_{p}\left(z_{n}, v\right)\right)^{\frac{1}{p^{2}-p}} \\
& =\frac{p^{\frac{1}{p^{2}-p}}}{\|A\|^{p}}\left(\frac{1}{q}\left(\left\|z_{n}\right\|^{p}-\left\|x_{n}\right\|^{p}\right)+\left\langle J_{E_{1}}^{p} z_{n}-J_{E_{1}}^{p} x_{n}, v\right\rangle\right)^{\frac{1}{p^{2}-p}}
\end{aligned}
$$

Therefore, we have that

$$
\lim _{n \rightarrow \infty}\left\|A x_{n}-\Pi_{A C_{n}}^{p} T^{n} A x_{n}\right\|=0 .
$$

By Lemma 2.11, we have that

$$
\lim _{n \rightarrow \infty} \triangle_{p}\left(A x_{n}, \Pi_{A C_{n}}^{p} T^{n} A x_{n}\right)=0 .
$$

On the other hand, since

$$
\begin{aligned}
& \triangle_{p}\left(y_{n}, x_{n}\right)=\triangle_{p}\left(y_{n}, x_{n+1}\right)+\Delta_{p}\left(x_{n+1}, x_{n}\right)-\left\langle y_{n}-x_{n+1}, J^{p} x_{n}-J^{p} x_{n+1}\right\rangle \\
& \leq\left[1+k_{n}\right] \triangle_{p}\left(z_{n}, x_{n+1}\right)+\left\langle J^{p} x_{n+1}-J^{p} x_{n}, x_{n+1}-x_{n},\right\rangle \\
& -\left\langle z_{n}-x_{n+1}, J^{p} x_{n}-J^{p} x_{n+1}\right\rangle+\mu_{n} \\
& \leq\left[1+k_{n}\right] \triangle_{p}\left(x_{n}, x_{n+1}\right)+\left\langle J^{p} x_{n+1}-J^{p} x_{n}, x_{n+1}-x_{n},\right\rangle \\
& -\left\langle z_{n}-x_{n+1}, J^{p} x_{n}-J^{p} x_{n+1}\right\rangle+\mu_{n} \\
& \rightarrow 0 \text { as } n \rightarrow \infty
\end{aligned}
$$

and

$$
\begin{aligned}
\triangle_{p}\left(y_{n}, z_{n}\right) & =\Delta_{p}\left(y_{n}, x_{n}\right)+\Delta_{p}\left(x_{n}, z_{n}\right) \\
& -\left\langle y_{n}-x_{n}, J^{p} z_{n}-J^{p} x_{n}\right\rangle \rightarrow 0 \text { as } n \rightarrow \infty .
\end{aligned}
$$

Now, from (52), we have that

$$
\begin{aligned}
& \Delta_{p}\left(y_{n}, v\right) \leq\left[k_{n}+1\right] \Delta_{p}\left(z_{n}, v\right)+\mu_{n} \\
& \quad\left(\alpha_{n}^{p}\left(1-\alpha_{n}\right)+\left(1-\alpha_{n}\right)^{p} \alpha_{n}\right) g\left(\left\|z_{n}-U^{n} z_{n}\right\|\right) .
\end{aligned}
$$

This implies, From (44), we have that

$$
\begin{aligned}
& g\left(\left\|z_{n}-U^{n} z_{n}\right\|\right) \leq \frac{\left[k_{n}+1\right] \triangle_{p}\left(z_{n}, v\right)+\mu_{n}-\Delta_{p}\left(y_{n}, v\right)}{\left(\alpha_{n}^{p}\left(1-\alpha_{n}\right)+\left(1-\alpha_{n}\right)^{p} \alpha_{n}\right)} \\
& =\frac{\frac{1}{q}\left(\left[k_{n}+1\right]\left\|z_{n}\right\|^{p}-\left\|y_{n}\right\|^{p}\right)+\left\langle J^{p} y_{n}-J^{p} z_{n}, v\right\rangle-k_{n}\left\langle J^{p} z_{n}, v\right\rangle}{\left(\alpha_{n}^{p}\left(1-\alpha_{n}\right)+\left(1-\alpha_{n}\right)^{p} \alpha_{n}\right)} . \\
& +\frac{\frac{k_{n}}{p}\|v\|^{p}+\mu_{n}}{\left(\alpha_{n}^{p}\left(1-\alpha_{n}\right)+\left(1-\alpha_{n}\right)^{p} \alpha_{n}\right)} .
\end{aligned}
$$

Since $\alpha_{n}\left(1-\alpha_{n}\right)>0$, and $g$ is continuous, strictly increasing and convex function, then we have that

$$
\lim _{n \rightarrow \infty} g\left\|z_{n}-U^{n}\left(z_{n}\right)\right\|=0
$$


and

$$
\lim _{n \rightarrow \infty}\left\|z_{n}-U^{n}\left(z_{n}\right)\right\|=0 .
$$

By Lemma 2.11, we have that

$$
\lim _{n \rightarrow \infty} \triangle_{p}\left(z_{n}, U^{n} z_{n}\right)=0 .
$$

Since $\mathrm{T}$ and $\mathrm{U}$ are continuous, then from (64) and (68), we have that

$$
\begin{aligned}
& \lim _{n \rightarrow \infty} \triangle_{p}\left(A x_{n}, \Pi_{A C}^{p} T A x_{n}\right)=0 \\
& \text { and } \\
& \quad \lim _{n \rightarrow \infty} \triangle_{p}\left(z_{n}, U z_{n}\right)=0 .
\end{aligned}
$$

Step five: we will show that $\left\{x_{n}\right\}$ converges strongly to an element of $\Gamma$. Since $\left\{x_{n}\right\}$ is Cauchy sequence, we may assume that $x_{n} \longrightarrow x^{*}$, from (61) we have $z_{n} \longrightarrow x^{*}$, which implies that $z_{n} \rightarrow x^{*}$. So it follows from (70) and the demicloseness of $(I-U)$ at zero that $x^{*} \in F i x(U)$. In addition, since $\mathrm{A}$ is bounded linear operator, we have that $\lim _{n \rightarrow \infty}\left\|A x_{n}-A x^{*}\right\|=0$. Hence, it follows from (69), and demicloseness of $\left(I-\Pi_{A C}^{p} T\right)$ at zero that $A x^{*} \in F i x\left(\Pi_{A C}^{p} T\right)$. This means that $x^{*} \in \Gamma$ and $\left\{x_{n}\right\}$ converges strongly to $x^{*} \in \Gamma$. The proof is completed.

In theorem 3.1, as $U=T, E_{1}=E_{2}=E$ and $A=I$, we have the following result.

Corollary 3.1.1. Let $E$ be a uniformly convex and uniformly smooth Banach spaces, $T: E \longrightarrow E$ be a uniformly continuous Bregman generalized asymptotically nonexpansive operator with the sequences $\left\{k_{n}\right\} \subset[0, \infty)$ and $\left\{\mu_{n}\right\} \subset[0, \infty)$ satisfying $\lim _{n \rightarrow \infty} k_{n}=0$ and $\lim _{n \rightarrow \infty} \mu_{n}=0$, respectively, and, for $p, q \in(1, \infty)$, $\Pi_{C}^{p}: E \longrightarrow C$ be a Bregman projection onto a subset $C \in E$, and $F i x(T) \neq \phi$ and $(I-T)$ be demiclosed at zero. Let $x_{1} \in E$ be chosen arbitrarily and let $C_{1}=E$. Define a sequence $\left\{x_{n}\right\}$ as follows;

$$
\left\{\begin{array}{l}
z_{n}=J_{E_{1}^{*}}^{q}\left(J_{E_{1}}^{p} x_{n}-\lambda_{n} J_{E_{2}}^{p}\left(I-\Pi_{C_{n}}^{p} T^{n}\right) x_{n}\right), \\
y_{n}=J_{E_{1}^{*}}^{q}\left(\alpha_{n} J_{E_{1}}^{p}\left(z_{n}\right)+\left(1-\alpha_{n}\right) J_{E_{1}}^{p}\left(T^{n}\left(z_{n}\right)\right)\right), \\
C_{n+1}=\left\{v \in C_{n}: \triangle_{p}\left(y_{n}, v\right) \leq\left[k_{n}+1\right] \triangle_{p}\left(z_{n}, v\right)+\mu_{n} ;\right. \\
\left.\triangle_{p}\left(z_{n}, v\right) \leq \triangle_{p}\left(x_{n}, v\right)\right\}, \\
x_{n+1}=\Pi_{C_{n+1}}^{p}\left(x_{1}\right), n \geq 1
\end{array} ;\right.
$$

where

$$
\lambda_{n}=\left\{\begin{array}{l}
\frac{1}{\left\|J_{E^{2}}^{p}\left(I-\Pi_{C_{n}}^{p} T^{n}\right) x_{n}\right\|}, x_{n} \neq 0 \\
\frac{\left\langle J_{E_{2}}^{2}\left(I-\Pi_{C_{n}^{n}}^{p}\right) x_{n}, x_{n}-\Pi_{C_{n}}^{p} T^{n} x_{n}\right\rangle^{p-1}}{\left\|J_{E_{2}}^{p}\left(I-\Pi_{C_{n}}^{p} T^{n}\right) x_{n}\right\|^{p}}, x_{n}=0
\end{array}\right.
$$

and $\gamma \in(0,1)$ and $\tau_{n}=\frac{1}{\left\|x_{n}\right\|^{p-1}}$ are chosen such that

$$
\rho_{E_{1}^{*}}\left(\tau_{n}\right)=\frac{\gamma}{{ }^{q} G_{q}} \times \frac{\left\langle J_{E_{2}}^{p}\left(I-\Pi_{C_{n}}^{p} T^{n}\right) x_{n}, x_{n}-\Pi_{C_{n}}^{p} T^{n} x_{n}\right\rangle}{\left\|x_{n}\right\|\left\|^{p}\right\| J_{E_{2}}^{p}\left(I-\Pi_{C_{n}}^{p} T^{n}\right) x_{n} \|},
$$

and $\left\{\alpha_{n}\right\} \subset(0,1)$ satisfies $\liminf _{n \rightarrow \infty} \alpha_{n}\left(1-\alpha_{n}\right)>0$. If $\operatorname{Fix}(T)=$ $\{v \in E: v=T v\} \neq \phi$, then $\left\{x_{n}\right\}$ converges strongly to $x^{*} \in$ $\operatorname{Fix}(T)$.

In theorem 3.1, when $\mathrm{U}$ and $\mathrm{T}$ are two Bregman symptotically nonexpansive mappings, the following result holds.

Corollary 3.1.2. Let $E_{1}$ and $E_{2}$ be two uniformly convex and uniformly smooth Banach spaces, $A: E_{1} \longrightarrow E_{2}$ is bounded and linear operator, such that $A(C)$, for $C \subset E_{1}$, is closed and convex, $U: E_{1} \longrightarrow E_{1}$ be a uniformly continuous Bregman asymptotically nonexpansive operator with the sequence $\left\{k_{n}^{(1)}\right\} \subset[1, \infty)$ satisfying $\lim _{n \rightarrow \infty} k_{n}^{(1)}=1$, and $T: E_{2} \longrightarrow$ $E_{2}$ be a uniformly continuous Bregman asymptotically nonexpansive operator with the sequence $\left\{k_{n}^{(2)}\right\} \subset[1, \infty)$ satisfying $\lim _{n \rightarrow \infty} k_{n}^{(2)}=1$, respectively and, for $p, q \in(1, \infty), \Pi_{A C}^{p}$ : $E_{2} \longrightarrow A C$ be a Bregman projection onto a subset $A C$, and $\operatorname{Fix}(U) \neq \phi$ and $\operatorname{Fix}\left(\Pi_{A C}^{p} T\right) \neq \phi$ respectively, and $(I-U)$ and $\left(I-\Pi_{A C}^{p} T\right)$ be demiclosed at zero. Let $x_{1} \in E_{1}$ be chosen arbitrarily and let $C_{1}=E_{1}$. Define a sequence $\left\{x_{n}\right\}$ as follows;

$$
\left\{\begin{array}{l}
z_{n}=J_{E_{1}^{*}}^{q}\left(J_{E_{1}}^{p} x_{n}-\lambda_{n} A^{*} J_{E_{2}}^{p}\left(I-\Pi_{A C_{n}}^{p} T^{n}\right) A x_{n}\right), \\
y_{n}=J_{E_{1}^{*}}^{q}\left(\alpha_{n} J_{E_{1}}^{p}\left(z_{n}\right)+\left(1-\alpha_{n}\right) J_{E_{1}}^{p}\left(U^{n}\left(z_{n}\right)\right)\right), \\
C_{n+1}=\left\{v \in C_{n}: \Delta_{p}\left(y_{n}, v\right) \leq k_{n} \triangle_{p}\left(z_{n}, v\right) \leq k_{n} \triangle_{p}\left(x_{n}, v\right)\right\} \\
x_{n+1}=\Pi_{C_{n+1}}^{p}\left(x_{1}\right), n \geq 1
\end{array}\right.
$$

where

$$
\lambda_{n}=\left\{\begin{array}{l}
\frac{1}{\|A\| \|} \frac{1}{\left\|J_{E_{2}}^{p}\left(I-\Pi_{A C_{n}}^{p} T^{n}\right) A x_{n}\right\|}, x_{n} \neq 0 \\
\frac{1}{\|A\|^{p}} \frac{\left\langle J_{E_{2}}^{p}\left(I-\Pi_{A C_{n}}^{p} T^{n}\right) A x_{n}, A x_{n}-\Pi_{A C_{n}}^{p} T^{n} A x_{n}\right\rangle^{p-1}}{\left\|J_{E_{2}}^{p}\left(I-\Pi_{A C_{n}}^{p} T^{n}\right) A x_{n}\right\|^{p}}, x_{n}=0
\end{array}\right.
$$

and $\gamma \in(0,1)$ and $\tau_{n}=\frac{1}{\left\|x_{n}\right\|^{p-1}}$ are chosen such that

$$
\rho_{E_{1}^{*}}\left(\tau_{n}\right)=\frac{\gamma}{2^{q} G_{q}\|A\|} \times \frac{\left\langle J_{E_{2}}^{p}\left(I-\Pi_{A C_{n}}^{p} T^{n}\right) A x_{n}, A x_{n}-\Pi_{A C_{n}}^{p} T^{n} A x_{n}\right\rangle}{\left\|x_{n}\right\|\left\|^{p}\right\| J_{E_{2}}^{p}\left(I-\Pi_{A C_{n}}^{p} T^{n}\right) A x_{n} \|},
$$

and $A^{*}$ denote the adjoint of $A$, and $\left\{\alpha_{n}\right\} \subset(0,1)$ satisfies $\liminf _{n \rightarrow \infty} \alpha_{n}\left(1-\alpha_{n}\right)>0, k_{n}=\left(k_{n}^{(1)} \vee k_{n}^{(2)}\right), n \geq 1$. If $\Gamma=\{v \in$ $\left.\operatorname{Fix}(U): A v \in F i x\left(\Pi_{A C}^{p} T\right)\right\} \neq \phi$, then $\left\{x_{n}\right\}$ converges strongly to $x^{*} \in \Gamma$.

In theorem 3.1, when $\mathrm{U}$ and $\mathrm{T}$ are two $\phi$-asymptotically nonexpansive mappings, the following result holds.

Corollary 3.1.3. Let $E_{1}$ and $E_{2}$ be two uniformly convex and uniformly smooth Banach spaces, $A: E_{1} \longrightarrow E_{2}$ is bounded and linear operator, such that $A(C)$, for $C \subset E_{1}$, is closed and convex, $U: E_{1} \longrightarrow E_{1}$ be a uniformly continuous $\phi$ asymptotically nonexpansive operator with the sequence $\left\{k_{n}^{(1)}\right\} \subset$ $[1, \infty)$ satisfying $\lim _{n \rightarrow \infty} k_{n}^{(1)}=1$, and $T: E_{2} \longrightarrow E_{2}$ be a uniformly continuous $\phi$-asymptotically nonexpansive operator with the sequence $\left\{k_{n}^{(2)}\right\} \subset[1, \infty)$ satisfying $\lim _{n \rightarrow \infty} k_{n}^{(2)}=1$, respectively and, for $p, q \in(1, \infty), \Pi_{A C}^{p}: E_{2} \longrightarrow A C$ be a generalized projection onto a subset $A C$, and $\operatorname{Fix}(U) \neq \phi$ and $F i x\left(\Pi_{A C}^{p} T\right) \neq \phi$ respectively, and $(I-U)$ and $\left(I-\Pi_{A C}^{p} T\right)$ be demiclosed at zero. Let $x_{1} \in E_{1}$ be chosen arbitrarily and let 
$C_{1}=E_{1}$. Define a sequence $\left\{x_{n}\right\}$ as follows;

$$
\left\{\begin{array}{l}
z_{n}=J_{E_{1}^{*}}^{-1}\left(J_{E_{1}} x_{n}-\lambda_{n} A^{*} J_{E_{2}}\left(I-\Pi_{A C_{n}}^{p} T^{n}\right) A x_{n}\right) \\
y_{n}=J_{E_{1}^{*}}^{-1}\left(\alpha_{n} J_{E_{1}}\left(z_{n}\right)+\left(1-\alpha_{n}\right) J_{E_{1}}\left(U^{n}\left(z_{n}\right)\right)\right), \\
C_{n+1}=\left\{v \in C_{n}: \phi\left(y_{n}, v\right) \leq k_{n} \phi\left(z_{n}, v\right) \leq k_{n} \phi\left(x_{n}, v\right)\right\} \\
x_{n+1}=\prod_{C_{n+1}}\left(x_{1}\right), n \geq 1
\end{array}\right.
$$

where

$$
\lambda_{n}=\left\{\begin{array}{l}
\frac{1}{\|A\|} \frac{1}{\left\|J_{E_{2}}\left(I-\Pi_{A C_{n}}^{p} T^{n}\right) A x_{n}\right\|}, \quad x_{n} \neq 0 \\
\frac{1}{\|A\|^{p}} \frac{\left\langle J_{E_{2}}\left(I-\Pi_{A C_{n}} T^{n}\right) A x_{n}, A x_{n}-\Pi_{A C_{n}} T^{n} A x_{n}\right\rangle}{\left\|J_{E_{2}}\left(I-\Pi_{A C_{n}} T^{n}\right) A x_{n}\right\|}, x_{n}=0
\end{array}\right.
$$

and $\gamma \in(0,1)$ and $\tau_{n}=\frac{1}{\left\|x_{n}\right\|}$ are chosen such that

$$
\rho_{E_{1}^{*}}\left(\tau_{n}\right)=\frac{\gamma}{2 G\|A\|} \times \frac{\left\langle J_{E_{2}}\left(I-\Pi_{A C_{n}} T^{n}\right) A x_{n}, A x_{n}-\Pi_{A C_{n}} T^{n} A x_{n}\right\rangle}{\left\|x _ { n } \left|\left\|\mid J_{E_{2}}\left(I-\Pi_{A C_{n}} T^{n}\right) A x_{n}\right\|\right.\right.},
$$

and $A^{*}$ denote the adjoint of $A$, and $\left\{\alpha_{n}\right\} \subset(0,1)$ satisfies $\liminf _{n \rightarrow \infty} \alpha_{n}\left(1-\alpha_{n}\right)>0, k_{n}=\left(k_{n}^{(1)} \vee k_{n}^{(2)}\right), n \geq 1$. If $\Gamma=\{v \in$ $\left.\underset{n \rightarrow \infty}{\operatorname{Fix}}(U): A v \in \operatorname{Fix}\left(\Pi_{A C} T\right)\right\} \neq \phi$, then $\left\{x_{n}\right\}$ converges strongly to $x^{*} \in \Gamma$.

In theorem 3.1, when $E_{1}$ and $E_{2}$ are real Hilbert spaces $H_{1}$ and $\mathrm{H}_{2}$, respectively, we have the following result.

Corollary 3.1.4. Let $H_{1}$ and $H_{2}$ be two real Hilbert spaces, $A$ : $H_{1} \longrightarrow H_{2}$ is bounded and linear operator, $U: H_{1} \longrightarrow H_{1}$ be a uniformly continuous generalized asymptotically nonexpansive operator with the sequences $\left\{k_{n}^{(1)}\right\} \subset[0, \infty)$ and $\left\{\mu_{n}^{(1)}\right\} \subset[0, \infty)$ satisfying $\lim _{n \rightarrow \infty} k_{n}^{(1)}=0$ and $\lim _{n \rightarrow \infty} \mu_{n}^{(1)}=0$, respectively, and $T$ : $\mathrm{H}_{2} \longrightarrow \mathrm{H}_{2}$ be a uniformly continuous generalized asymptotically nonexpansive operator with the sequences $\left\{k_{n}^{(2)}\right\} \subset[0, \infty)$ and $\left\{\mu_{n}^{(2)}\right\} \subset[0, \infty)$ satisfying $\lim _{n \rightarrow \infty} k_{n}^{(2)}=0$ and $\lim _{n \rightarrow \infty} \mu_{n}^{(2)}=0$, respectively, and $(I-U)$ and $(I-T)$ be demiclosed at zero. Let $x_{1} \in H_{1}$ be chosen arbitrarily and let $C_{1}=H_{1}$ and the sequence $\left\{x_{n}\right\}$ be defined as follows;

$$
\left\{\begin{array}{l}
\left.z_{n}=x_{n}-\lambda_{n} A^{*}\left(I-T^{n}\right) A x_{n}\right), \\
y_{n}=\alpha_{n} z_{n}+\left(1-\alpha_{n}\right) U^{n}\left(z_{n}\right), \\
C_{n+1}=\left\{v \in C_{n}:\left\|y_{n}-v\right\| \leq\left[k_{n}+1\right]\left\|z_{n}-v\right\|+\mu_{n}\right. \\
\left.\left\|z_{n}-v\right\| \leq\left[k_{n}+1\right]\left\|x_{n}-v\right\|+\mu_{n}\right\}, \\
x_{n+1}=P_{C_{n+1}}\left(x_{1}\right), n \geq 1
\end{array}\right.
$$

where $\lambda_{n} \in\left(0, \frac{1}{\left\|A^{*}\right\|^{2}}\right)$ and $A^{*}$ denote the adjoint of $A$, and $\left\{\alpha_{n}\right\} \subset$ $(0,1)$ satisfies $\liminf _{n \rightarrow \infty} \alpha_{n}\left(1-\alpha_{n}\right)>0$, and $k_{n}=\left(k_{n}^{(1)} \vee k_{n}^{(2)}\right), n \geq 1$. If $\Gamma=\{v \in F i x(U): A v \in F i x(T)\} \neq \phi$, then $\left\{x_{n}\right\}$ converges strongly to $x^{*} \in \Gamma$.

In theorem 3.1, when $\mathrm{U}$ and $\mathrm{T}$ are two nonexpansive mappings, the following result holds.

Corollary 3.1.5. Let $E_{1}$ and $E_{2}$ be two uniformly convex and uniformly smooth Banach spaces, $A: E_{1} \longrightarrow E_{2}$ is bounded and linear operator, such that $A(C)$, for $C \subset E_{1}$, is closed and convex, $U: E_{1} \longrightarrow E_{1}$ be a uniformly continuous Bregman nonexpansive operator, and $T: E_{2} \longrightarrow E_{2}$ be a uniformly continuous Bregman nonexpansive operator, and, for $p, q \in(1, \infty)$,
$\Pi_{A C}^{p}: E_{2} \longrightarrow A C$ be a Bregman projection onto a subset $A C$, and $F i x(U) \neq \phi$ and $F i x\left(\Pi_{A C}^{p} T\right) \neq \phi$ respectively, and $(I-U)$ and $\left(I-\Pi_{A C}^{p} T\right)$ be demiclosed at zero. Let $x_{1} \in E_{1}$ be chosen arbitrarily and let $C_{1}=E_{1}$ and the sequence $\left\{x_{n}\right\}$ be defined as follows;

$$
\left\{\begin{array}{l}
z_{n}=J_{E_{1}^{*}}^{q}\left(J_{E_{1}}^{p} x_{n}-\lambda_{n} A^{*} J_{E_{2}}^{p}\left(I-\Pi_{A C_{n}}^{p} T\right) A x_{n}\right), \\
y_{n}=J_{E_{1}^{*}}^{q}\left(\alpha_{n} J_{E_{1}}^{p}\left(z_{n}\right)+\left(1-\alpha_{n}\right) J_{E_{1}}^{p}\left(U\left(z_{n}\right)\right)\right), \\
C_{n+1}=\left\{v \in C_{n}: \Delta_{p}\left(y_{n}, v\right) \leq \Delta_{p}\left(z_{n}, v\right) \leq \Delta_{p}\left(x_{n}, v\right)\right\} \\
x_{n+1}=\Pi_{C_{n+1}}^{p}\left(x_{1}\right), n \geq 1
\end{array}\right.
$$

where

$$
\lambda_{n}=\left\{\begin{array}{l}
\frac{1}{\|A\|} \frac{1}{\left\|J_{E_{2}}^{p}\left(I-\Pi_{A C_{n}}^{p} T\right) A x_{n}\right\|}, x_{n} \neq 0 \\
\frac{1}{\|A\|^{p}} \frac{\left\langle J_{E_{2}}^{p}\left(I-\Pi_{A C_{n}}^{p} T\right) A x_{n}, A x_{n}-\Pi_{A C_{n}}^{p} T A x_{n}\right\rangle^{p-1}}{\left\|J_{E_{2}}^{p}\left(I-\Pi_{A C_{n}}^{p} T\right) A x_{n}\right\|^{p}}, x_{n}=0
\end{array}\right.
$$

and $\gamma \in(0,1)$ and $\tau_{n}=\frac{1}{\left\|x_{n}\right\|^{p-1}}$ are chosen such that

$$
\rho_{E_{1}^{*}}\left(\tau_{n}\right)=\frac{\gamma}{2^{q} G_{q}\|A\|} \times \frac{\left\langle J_{E_{2}}^{p}\left(I-\Pi_{A C_{n}}^{p} T\right) A x_{n}, A x_{n}-\Pi_{A C_{n}}^{p} T A x_{n}\right\rangle}{\left\|x_{n}\right\|^{p}\left\|J_{E_{2}}^{p}\left(I-\Pi_{A C_{n}}^{p} T\right) A x_{n}\right\|},
$$

and $A^{*}$ denote the adjoint of $A$, and $\left\{\alpha_{n}\right\} \subset(0,1)$ satisfies $\liminf _{n \rightarrow \infty} \alpha_{n}\left(1-\alpha_{n}\right)>0$. If $\Gamma=\left\{v \in F i x(U): A v \in F i x\left(\Pi_{A C}^{p} T\right)\right\} \neq$ $\phi$, then $\left\{x_{n}\right\}$ converges strongly to $x^{*} \in \Gamma$.

\section{Application to the mixed equilibrium problem}

Lemma 4.1. [4] Let E be a reflexive, strictly convex and smooth Banach space, and let $C$ be a nonempty closed convex subset of E. Let $f, g: C \times C \longrightarrow \mathfrak{R}$ be two bifunctions which satisfy the conditions $\left(A_{1}\right)-\left(A_{4}\right),\left(B_{1}\right)-\left(B_{3}\right)$ and $(C)$, in $(6)$, then for every $x \in E$ and $r>0$, there exists a unique point $z \in C$ such that

$$
\left.f(z, y)+g(z, y)+\frac{1}{r}\langle y-z, j z-j x\rangle \geq 0 \forall y \in C\right\}
$$

In Reich, S, Sabach, S (2010) [18], when $f(x)=\frac{1}{p}\|x\|^{p}$ then we have the following Lemma.

Lemma 4.2. Let $E$ be a reflexive, strictly convex and smooth Banach space, and let $C$ be a nonempty closed convex subset of E. Let $f, g: C \times C \longrightarrow \mathfrak{R}$ be two bifunctions which satisfy the conditions $\left(A_{1}\right)-\left(A_{4}\right),\left(B_{1}\right)-\left(B_{3}\right)$ and $(C)$, in (6), then for every $x \in E$ and $r>0$, define a mapping $S_{r}: E \longrightarrow C$ as follows;

$S_{r}(x)=\left\{x \in C: f(z, y)+g(z, y)+\frac{1}{r}\left\langle y-z, J_{E}^{p} z-J_{E}^{p} x\right\rangle \geq 0 \forall y \in C\right\}$

then the following hold;

1. $S_{r}$ is a single-valued;

2. $S_{r}$ is a Bregman firmly nonexpansive-type mapping, i.e.

$\forall x, y \in E\left\langle S_{r} x-S_{r} y, J_{E}^{p} S_{r} x-J_{E}^{p} S_{r} y\right\rangle \leq\left\langle S_{r} x-S_{r} y, J_{E}^{p} x-J_{E}^{p} y\right\rangle ;$ or equivalently $\triangle_{p}\left(S_{r} x, S_{r} y\right)+\triangle_{p}\left(S_{r} y, S_{r} x\right)+\Delta_{p}\left(S_{r} x, x\right)+\triangle_{p}\left(S_{r} y, y\right) \leq$ $\triangle_{p}\left(S_{r} x, y\right)+\triangle_{p}\left(S_{r} y, x\right)$ 
3. $F\left(S_{r}\right)=\operatorname{MEP}(f, g)$;

4. $\operatorname{MEP}(f, g)$ is closed and convex;

5. for all $x \in E$ and for all $v \in F\left(S_{r}\right), \Delta_{p}\left(v, S_{r} x\right)+\triangle_{p}\left(S_{r} x, x\right) \leq$ $\triangle_{p}(v, x)$.

Theorem 4.1. Let $E_{1}$ and $E_{2}$ be two uniformly convex and uniformly smooth Banach spaces, $A: E_{1} \longrightarrow E_{2}$ is bounded and linear operator, such that $A(C)$, for $C \subset E_{1}$, is closed and convex, $T: E_{2} \longrightarrow E_{2}$ be a uniformly continuous Bregman nonexpansive operator, and, for $p, q \in(1, \infty), \Pi_{A C}^{p}: E_{2} \longrightarrow A C$ be a Bregman projection onto a subset $A C$, and $f, g: C \times C \longrightarrow \mathfrak{R}$ be two bifunctions which satisfy the conditions $\left(A_{1}\right)-\left(A_{4}\right),\left(B_{1}\right)-$ $\left(B_{3}\right)$ and $(C)$, in (6), for $C \times C \subset E_{1} \times E_{1}$, assume that $C=$ $\operatorname{MEP}(f, g) \neq \phi$ and $\operatorname{Fix}(T) \neq \phi$, taking $C_{1}=E_{1}$, the sequence $\left\{x_{n}\right\}$ is defined as follows;

$$
\left\{\begin{array}{l}
\text { choosing arbitrarily } x_{1} \in E_{1}, \\
z_{n}=J_{E_{1}^{*}}^{q}\left(J_{E_{1}}^{p} x_{n}-\lambda_{n} A^{*} J_{E_{2}}^{p}\left(I-\Pi_{A C_{n}}^{p} T^{n}\right) A x_{n}\right), \\
f\left(S_{r}\left(x_{n}\right), b\right)+g\left(S_{r}\left(x_{n}\right), b\right)+\frac{1}{r_{n}}\left\langle b-S_{r}\left(x_{n}\right), J_{E_{1}}^{p}\left(S_{r}\left(x_{n}\right)\right)\right. \\
\left.-J_{E_{1}}^{p}\left(x_{n}\right)\right\rangle \geq 0 \forall b \in E_{1} \\
y_{n}=J_{E_{1}^{*}}^{q}\left(\alpha_{n} J_{E_{1}}^{p}\left(z_{n}\right)+\left(1-\alpha_{n}\right) J_{E_{1}}^{p}\left(S_{r}\left(z_{n}\right)\right)\right) \\
C_{n+1}=\left\{v \in C_{n}: \Delta_{p}\left(y_{n}, v\right) \leq \Delta_{p}\left(z_{n}, v\right) \leq \Delta_{p}\left(x_{n}, v\right)\right\} \\
x_{n+1}=\Pi_{C_{n+1}}^{p}\left(x_{1}\right), n \geq 1,
\end{array}\right.
$$

where

$$
\lambda_{n}=\left\{\begin{array}{l}
\frac{1}{\|A\|} \frac{1}{\left\|J_{E_{2}^{p}}^{p}\left(I-\Pi_{A C_{1}^{p}}^{p} T^{n}\right) A x_{n}\right\|}, x_{n} \neq 0 \\
\frac{1}{\|A\|^{p}} \frac{\left\langle J_{E_{2}}^{p}\left(I-\Pi_{A C_{n}}^{p} T^{n}\right) A x_{n}, A x_{n}-\Pi_{A C_{n}}^{p} T^{n} A x_{n}\right)^{p-1}}{\left\|J_{E_{2}}^{p}\left(I-\Pi_{A C_{n}}^{p} T^{n}\right) A x_{n}\right\|^{p}}, x_{n}=0
\end{array}\right.
$$

and $\gamma \in(0,1)$ and $\tau_{n}=\frac{1}{\left\|x_{n}\right\|^{p-1}}$ are chosen such that

$$
\rho_{E_{1}^{*}}\left(\tau_{n}\right)=\frac{\gamma}{2^{q} G_{q}\|A\|} \times \frac{\left\langle J_{E_{2}}^{p}\left(I-\Pi_{A C_{n}}^{p} T^{n}\right) A x_{n}, A x_{n}-\Pi_{A C_{n}}^{p} T^{n} A x_{n}\right\rangle}{\left\|x_{n}\right\|^{p}\left\|J_{E_{2}}^{p}\left(I-\Pi_{A C_{n}}^{p} T^{n}\right) A x_{n}\right\|},
$$

and $A^{*}$ denote the adjoint of $A$, and $\left\{\alpha_{n}\right\} \subset(0,1)$ satisfies $\liminf _{n \rightarrow \infty} \alpha_{n}\left(1-\alpha_{n}\right)>0$. If $\Gamma=\left\{v \in M E P(f, g): A v \in \operatorname{Fix}\left(\Pi_{A C}^{p} T\right)\right\} \neq$ $\phi$, then $\left\{x_{n}\right\}$ converges strongly to $x^{*} \in \Gamma$.

Proof: It follows from the lemma 4.2 that $\operatorname{Fix}\left(S_{r}\right)=$ $\operatorname{MEP}(f, g)$ is nonempty, closed and convex and $S_{r}$ is a firmly nonexpansive mapping. Meanwhile $S_{r}$ is assume to be continuous. Hence all conditions in corollary 3.1.5 are satisfied. The conclusion of theorem 4.1 can be directly obtained from corollary 3.1.5.

Let $E_{1}$ and $E_{2}$ be two uniformly convex and uniformly smooth Banach spaces. Let $\mathrm{C}$ and $\mathrm{Q}$ be nonempty closed convex subsets of $E_{1}$ and $E_{2}$, respectively. $A: E_{1} \longrightarrow E_{2}$ is bounded and linear operator. Assume that $f, g: C \times C \longrightarrow \mathfrak{R}$ be two bifunctions and $f^{\prime}, g^{\prime}: Q \times Q \longrightarrow \mathfrak{R}$ be another two bifunctions. The split mixed equilibrium problem (SMEP) is to find an element $v \in C$ such that

$$
f(v, y)+g(v, y) \geq 0 \forall y \in C
$$

and such that $A v \in Q$ solve

$$
f(A v, A y)+g(A v, A y) \geq 0 \forall A y \in Q
$$

Let $\omega=\left\{v \in M E P(f, g): A v \in M E P\left(f^{\prime}, g^{\prime}\right)\right\}$ denote the solution set of the SMEP.

Corollary 4.1.1. Let $E_{1}$ and $E_{2}$ be two uniformly convex and uniformly smooth Banach spaces. Let $C$ and $Q$ be nonempty closed convex subsets of $E_{1}$ and $E_{2}$, respectively. $A: E_{1} \longrightarrow$ $E_{2}$ is bounded and linear operator, such that $A(C)$ is closed and convex, and, such that $A(C)$, for $C \subset E_{1}$, is closed and convex, for $p, q \in(1, \infty), \Pi_{A C}^{p}: E_{2} \longrightarrow A C$ be a Bregman projection onto a subset AC. Assume that $f, g: C \times C \longrightarrow$ $\mathfrak{R}$ be two bifunctions and $f^{\prime}, g^{\prime}: Q \times Q \longrightarrow \mathfrak{R}$ be another two bifunctions which satisfy the conditions $\left(A_{1}\right)-\left(A_{4}\right),\left(B_{1}\right)-$ $\left(B_{3}\right)$ and $(C)$, in (6), for $C \times C \subset E_{1} \times E_{1}$, assuming $M E P(f, g) \neq$ $\phi$ and $\operatorname{MEP}\left(\Pi_{A C}^{p}, f^{\prime}, g^{\prime}\right) \neq \phi$, respectively, taking $C_{1}=E_{1}$, the sequence $\left\{x_{n}\right\}$ is defined as follows;

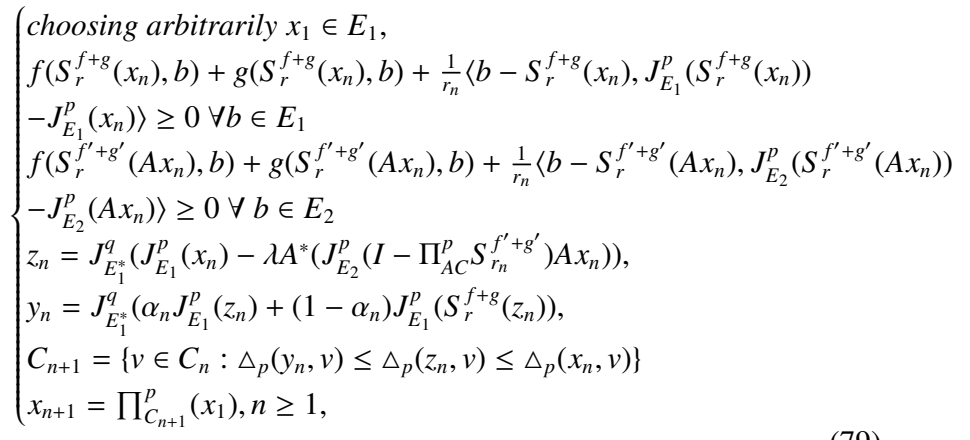

where

$$
\lambda_{n}=\left\{\begin{array}{l}
\frac{1}{\|A\|} \frac{1}{\left\|J_{E_{2}}^{p}\left(I-\Pi_{A}^{p} S_{r_{n}^{\prime}}^{f^{\prime}+g^{\prime}}\right) A x_{n}\right\|}, x_{n} \neq 0 \\
\frac{1}{\|A\|^{p}} \frac{\left.\left\langle J_{E_{2}}^{p}\left(I-\Pi_{A C}^{p} S_{r_{n}}^{f^{\prime}+g^{\prime}}\right) A x_{n}\right), A x_{n}-\Pi_{A C}^{p} S_{r_{n}}^{f^{\prime}+g^{\prime}} A x_{n}\right\rangle^{p-1}}{\left\|J_{E_{2}}^{p}\left(I-\Pi_{A C}^{p} S_{r_{n}}^{f^{\prime}+g^{\prime}}\right) A x_{n}\right\|^{p}}, x_{n}=0
\end{array}\right.
$$

and $\gamma \in(0,1)$ and $\tau_{n}=\frac{1}{\left\|x_{n}\right\|^{p-1}}$ are chosen such that

$\rho_{E_{1}^{*}}\left(\tau_{n}\right)=\frac{\gamma}{2^{q} G_{q}\|A\|} \frac{\left\langle J_{E_{2}}\left(I-\Pi_{A C}^{p} S_{r_{n}}^{f^{\prime}+g^{\prime}}\right) A x_{n}, A x_{n}-\Pi_{A C}^{p} S_{r_{n}}^{f^{\prime}+g^{\prime}} A x_{n}\right\rangle}{\left\|x_{n}\right\|^{p}\left\|J_{E_{2}}^{p}\left(I-\Pi_{A C}^{p} S_{r_{n}}^{f^{\prime}+g^{\prime}}\right) A x_{n}\right\|}$

and $A^{*}$ denote the adjoint of $A$, and $\left\{r_{n}\right\} \subset(0,1)$, and $\left\{\alpha_{n}\right\} \subset$ $(0,1)$ satisfies $\lim _{n \rightarrow \infty} \alpha_{n}\left(1-\alpha_{n}\right)>0, n \geq 1$. If $\omega=\{v \in$ $\left.\operatorname{MEP}(f, g): A v \in \operatorname{MEP}\left(\Pi_{A C}^{p}, f^{\prime}, g^{\prime}\right)\right\} \neq \phi$, then $\left\{x_{n}\right\}$ converges strongly to $x^{*} \in \omega$.

\section{An Example}

In this section we discuss how to apply Theorem 3.1 on the following example.

Let $E_{1}=E_{2}=C_{1}=(-\infty, \infty), A=A^{*}=I, p=q=2$, and $\alpha_{n}=\frac{1}{2^{n}}$. Define

$$
U: E_{1} \longrightarrow E_{1} \text { by } U(x)=\left\{\begin{array}{l}
x, x \in(-\infty, 0]=B_{1} \\
\frac{1}{2} x, x \in(0, \infty)=B_{2},
\end{array}\right.
$$$$
A: E_{1} \longrightarrow E_{2} \text { by } A(x)=x, x \in(-\infty, \infty) \text {, }
$$ 


$$
\begin{aligned}
& T: E_{2} \longrightarrow E_{2} \text { by } T(x)=\left\{\begin{array}{l}
x, x \in(-\infty, 0]=D_{1} \\
\frac{1}{4} x, x \in(0, \infty)=D_{2},
\end{array}\right. \\
& \Pi_{C}^{2}: E_{2} \longrightarrow C \text { by } \Pi_{C}^{2}(x)=\left\{\begin{array}{l}
0, x \in(-\infty, 0) \\
x, x \in[0, \infty),
\end{array}\right. \\
& \Pi_{C}^{2} T: E_{2} \longrightarrow \text { C by } \Pi_{C}^{2} T(x)=\left\{\begin{array}{l}
x, x \in\{0\} \\
0, x \in(-\infty, 0) \\
\frac{1}{4} x, x \in(0, \infty)
\end{array}\right.
\end{aligned}
$$

Now we check whether or not the mappings $U$ and $T$ are uniformly continuous Bregman generalized asymptotically nonexpansive. To do this it suffices to check for each map the following four cases.

Case I: if $x, y \in B_{1}$ then

$$
\triangle_{2}\left(U^{n}(x), U^{n}(y)\right)=\Delta_{2}(x, y) \leq\left[\frac{1}{2^{n}}+1\right] \Delta_{2}(x, y)+\frac{1}{2^{n}} ;
$$

Case II: if $x, y \in B_{2}$ then

$$
\begin{aligned}
\triangle_{2}\left(U^{n}(x), U^{n}(y)\right) & =\triangle_{2}\left(\frac{1}{2^{n}} x, \frac{1}{2^{n}} y\right) \\
& =\frac{1}{2^{2 n}} \triangle_{2}(x, y) \leq\left[\frac{1}{2^{n}}+1\right] \triangle_{2}(x, y)+\frac{1}{2^{n}} ;
\end{aligned}
$$

Case III: if $x \in B_{2}$ and $y \in B_{1}$, then

$$
\begin{aligned}
& \triangle_{2}\left(U^{n}(x), U^{n}(y)\right)=\Delta_{2}\left(\frac{1}{2^{n}} x, y\right)=\frac{1}{2^{2 n}} \frac{1}{2}|x|^{2}-\frac{1}{2^{n}}\langle x, y\rangle+\frac{1}{2}|y|^{2} \\
& \leq \frac{1}{2}|x|^{2}-\langle x, y\rangle+\frac{1}{2}|y|^{2}=\Delta_{2}(x, y) \leq\left[\frac{1}{2^{n}}+1\right] \Delta_{2}(x, y)+\frac{1}{2^{n}}
\end{aligned}
$$

Case IV: if $x \in B_{1}$ and $y \in B_{2}$, then

$$
\begin{aligned}
& \triangle_{p}\left(U^{n}(x), U^{n}(y)\right)=\Delta_{2}\left(x, \frac{1}{2^{n}} y\right) \\
& \quad=\frac{1}{2}|x|^{2}-\frac{1}{2^{n}}\langle x, y\rangle+\frac{1}{2} \frac{1}{2^{2 n}}|y|^{2} \\
& \quad \leq \frac{1}{2}|x|^{2}-\langle x, y\rangle+\frac{1}{2}|y|^{2}=\Delta_{2}(x, y) \leq\left[\frac{1}{2^{n}}+1\right] \triangle_{2}(x, y)+\frac{1}{2^{n}} .
\end{aligned}
$$

Since it is clear that $U$ is uniformly continuous, then from (80), (81), (82) and (83) $U$ is a uniformly continuous Bregman generalized asymptotically nonexpansive mapping. Similarly, it is also clear that $T$ is a uniformly continuous Bregman generalized asymptotically nonexpansive mapping. Next, we simplify the scheme as follows.

Clearly $F i x(U)=(-\infty, 0], F i x(T)=(-\infty, 0]$ and $F i x\left(\Pi_{C}^{2} T\right)=$ $\{0\}$ such that $\Gamma=\left\{x \in F i x(U) ; A x \in F i x\left(\Pi_{C}^{2} T\right)\right\}=\{0\}$. Now we have that

$$
\lambda_{n}=\left\{\begin{array}{l}
\frac{1}{\left|x_{n}\right|}, x_{n} \in(-\infty, 0) \\
\frac{4}{3 x_{n}}, x_{n} \in(0, \infty) \\
1, x_{n} \in\{0\} .
\end{array}\right.
$$

$$
\begin{aligned}
& z_{n}=\left\{\begin{array}{l}
x_{n}+1, x_{n} \in(-\infty, 0) \\
x_{n}-1, x_{n} \in(0, \infty) \\
0, x_{n} \in\{0\}
\end{array}\right. \\
& y_{n}=\left\{\begin{array}{l}
x_{n}+1, x_{n} \in(-\infty, 0) \\
\left(\frac{2}{2^{n}}-\frac{1}{2^{2 n}}\right)\left(x_{n}-1\right), x_{n} \in(0, \infty) \\
0, x_{n} \in\{0\},
\end{array}\right.
\end{aligned}
$$$$
C_{n+1}=\left\{v \in C_{n} ;\left\{\begin{array}{l}
\frac{1}{2}\left|x_{n}+1-v\right|^{2} \leq\left[\frac{1}{2^{n}}+1\right] \frac{1}{2}\left|x_{n}+1-v\right|^{2}+\frac{1}{2^{n}} \\
\frac{1}{2}\left|x_{n}+1-v\right|^{2} \leq \frac{1}{2}\left|x_{n}-v\right|^{2}, x_{n} \in(-\infty, 0) \\
\frac{1}{2}\left|\left(\frac{2}{2^{n}}-\frac{1}{2^{2 n}}\right)\left(x_{n}-1\right)-v\right|^{2} \leq\left[\frac{1}{2^{n}}+1\right] \frac{1}{2}\left|x_{n}-1-v\right|^{2}+\frac{1}{2^{n}} \\
\frac{1}{2}\left|x_{n}-1-v\right|^{2} \leq \frac{1}{2}\left|x_{n}-v\right|^{2}, x_{n} \in(0, \infty) \\
\left.\frac{1}{2}|0-v|^{2} \leq\left[\frac{1}{2^{n}}+1\right] \frac{1}{2}|0-v|^{2}+\frac{1}{2^{n}}, x_{n} \in\{0\}\right\},
\end{array}\right.\right.
$$

Now, take the initial point $x_{1}=0.5$ from positive real numbers, the numerical experiment result is given below;

$z_{1}=-0.5 ; y_{1}=-0.375 ; C_{2}=(-\infty, 0] ; x_{2}=0 ;$

$z_{2}=0 ; y_{2}=0 ; x_{3}=x_{2}=0$.

This implies that zero is in the solution set $\Gamma$. Similarly, from the negative real numbers, let $x_{1}=-0.5$ then we arrive at the same fixed point zero as follows;

$z_{1}=0.5 ; y_{1}=0.5 ; C_{2}=[0, \infty) ; x_{2}=0 ;$

$z_{2}=0 ; y_{2}=0 ; x_{3}=x_{2}=0$.

\section{Conclusion}

In summary, the validity of the proposed scheme is provided by step one and two in the manuscript. Strong convergence of the proposed sequence is achieved in step three. Approximate fixed point sequence of the proposed mappings is given by step four. Application of the demicloseness principle for the proposed mappings is discussed in step five. Furthermore, in this paper, the strong convergence for SCFPP of Bregman generalized asymptotically nonexpansive mapping is obtained in uniformly convex and uniformly smooth Banach spaces, without the assumption of semi-compactness property and or without the assumption of Opial condition. This infer that, the main result presented here generalizes and extends that of Zhang et al. [10] and the references therein. This is true by the fact that the map presented here is more general than asymptotically nonexpansive map which was used by Zhang et. al. So also the space used here generalizes the one applied in the work of Zhang etal. Moreso, the notion of Bregman distance used here is the general case of the classical norm distance used in the result of Zhang et al.

\section{Acknowledgments}

We thank the referees for the positive enlightening comments and suggestions, which have greatly helped us in making improvements to this paper. 


\section{References}

[1] Y. Censor, T. Elfving,N. Kopf \& T. Bortfeld, "The multiple-sets split feasibility problem and its applications", Inverse Probl. 21 (2005) 2071.

[2] C. Byrne, "Iterative oblique projection onto convex subsets and the split feasibility problems", Inverse Probl. 18 (2002) 441.

[3] Y. Censor \& A. Segal, "The split common fixed point problem for directed operators", J. Convex Anal. 16 (2009) 587.

[4] B. C. Deng, T. Chen \& Y. L. Yin, "Strong convergence theorems for mixed equilibrium problem and asymptotically I-nonexpansive mapping in Banach spaces", Abstract and Applied Anal. 2014 (2014) Article ID 965737.

[5] L. Yang, S. S. Chang, Y. J. Cho \& J. K. Kim, Multiple-set split feasibility problems for total asymptotically strict pseudocontractions mappings, Fixed Point Theory Appl. 1 (2011) 77.

[6] B. Qu \& N. Xiu, "Note on the CQ algorithm for the split feasibility problem", Inverse Probl. 21 (2005) 1655.

[7] F. Schopfer, "Iterative Methods for the Solution of the Split Feasibility Problem in Banach Spaces", der Naturwissenschaftlich-Technischen Fakultaten, Universitat des Saarlandes, 2007.

[8] A. Moudafi, "The split common fixed point problem for demi-contractive mappings", Inverse Probl. 26 (2014).

[9] S. S. Chang, Y. J. Cho, J. K. Kim, W. Zhang \& L. Yang, "Multiple-set split feasibility problems for asymptotically strict pseudocontractions", Abstr. Appl. Anal. 2012 (2012) 491760.

[10] X. F. Zhang, L. Wang, Z. L. Ma \& L. J. Qin "The strong convergence the- orems for split common fixed point problem of asymptotically nonexpansive mappings in Hlibert spaces", Journal of Inequalities and Applications 1 (2015) 1.

[11] W. Takahashi, "The split common null point problem in Banach spaces", Arch. Math. (Basel) 104 (2015) 357.

[12] J. Tang, S. S. Chang, L. Wang \& X. Wang, "On the split common fixed point problem for strict pseudocontractive and asymptotically nonexpansive mappings in Banach spaces", Journal of Inequalities and Applications 2015 (2015) 305.

[13] J. A. Clarkson, "Uniformly convex spaces", Trans. Am. Math. Soc. 40 (1936) 396.

[14] C. E. Chidume, "Geometric properties in Banach spaces and nonlinear iterations", Springer-Verlag London Limited, 2009.

[15] F. Shopfer, T. Schuster and A. K. Louis, "Metri and Bregman projection onto affine subspaces and theircomputation via sequential subspace optimization methods", J. Inv. III-posed problem 15 (2007) 1.

[16] Z. B. Xu \& G. F. Roach, "Characteristic inequalities of uniformly convex and uniformly smooth Banach spaces", Journal of Mathematical Analysis and Applications, 1991.

[17] H. K. Xu, "Inequalities in Banach spaces with applications", Nomlinear Analysis 12 (1991) 1127.

[18] S. Reich and S. Sabach, "Two strong convergence theorems for Bregman strongly nonexpansive operators in reflexive Banach spaces", Nonlinear Anal. 73 (2010) 122. 